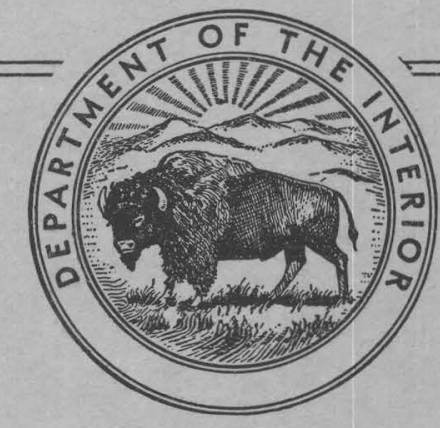

August 1949

\title{
INDEX OF SURFACE-WATER RECORDS
}

PART 10.-THE GREAT BASIN

TO SEPTEMBER 30,1948

Prepared by Salt Lake City District 


\section{UNITED STATES DEPARTMENT OF THE INTERIOR}

J. A. Krug, Secretary

GEOLOGICAL SURVEY

W. E. Wrather, Director

WASHINGTON, D. C.

Free on application to the Director, Geological Survey, Washington 25, D. C. 


\title{
INDEX OF SURFACE-WATER RECORDS
}

\author{
PART 10.-THE GREAT BASIN TO SEPTEMBER 30, 1948
}

\section{EXPLANATION}

The Index lists the stream-flow and reservoir stations in the Great Basin for which records have been or are to be published for periods prior to September 30, 1948. The stations are listed in downstream order. Tributary streams are indicated by indention.

Station names are given in their most recently published forms. Parentheses around part of a station name indicate that the inclosed word or words were used in an earlier published name of the station or in a name under which records were published by some agency other than the Geological Survey.

The drainage areas, in square miles, are the latest figures published or otherwise ava1lable at thls time. Drainage areas that were obviously inconsistent with other drainage areas on the same stream have been omitted.

Under pperlod of recordn breaks of less than a 12-month period are not shown. A desh not followed 1mmediately by a closing date shows that the station was in operation on September 30 , 1948. The yearg. given are calendar years. Perlods of records published by agencles other than the Geological survey are listed in parentheses only when they contain more detalled information or are for perlods not reported in publications of the Geological Survey.

Records both of gage helght and of discharge are listed for atream-flow stations, and records of gage holght and of contents (or of change in contents) are listed for stations on reservolrs. Records of gage belghts only and records consisting only of monthly figures elther of stream flor or reservolr contents are designated by aymbols and footnotes. For early years when da1ly discharges were not generally published by the Geological Survey, publiahed dally gage holghts and a publishod rating table were considered to be equivalent to dally discharges.

An alphabetical index of streams, canals, and reservolrs is given on pages 15 to 18. 
GREAT SALT LAKE BASIN

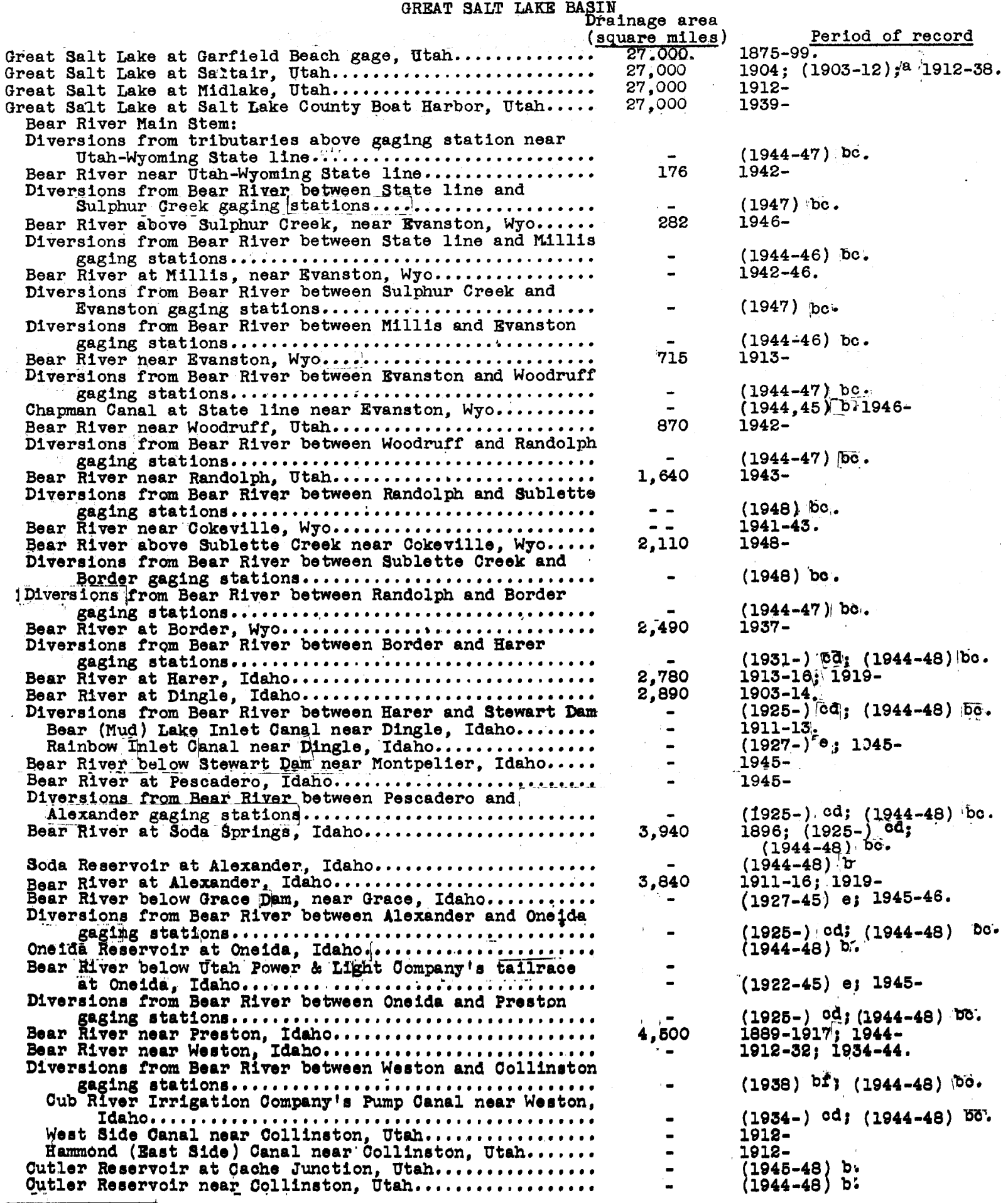

a In reports of United States Weather Burea'u.

b In Bear River Hydrometr10 Deta Tri-state Invest1gations Report.

- Irrigation season records only.

d In roports of Wator District No. 5 - Idaho.

- 1927-45 in f1les of Geological Surver at Salt Lake 01ty, Jtah.

$f$ In reports of state Bngineor of Utah. 
Great Salt Lake tributaries--Continued.

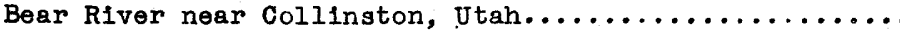

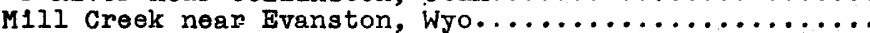

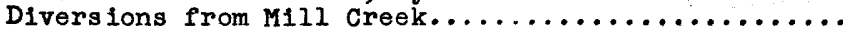

M111 Greek below diversions near Evanston, Wyo........ Sulphur Creek above diversions near Evanston, Wyo.....

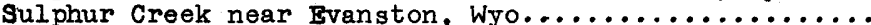
Willow Creek above diversions near Evanston, Wyo.... Diversions from Sulphur Creek and. Willow Creek......

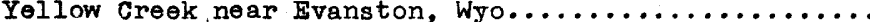
Yellow Creek below diversions near Evanston, Wyo......

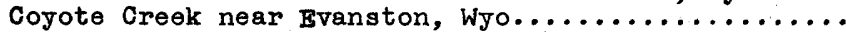

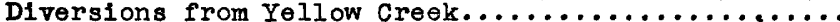

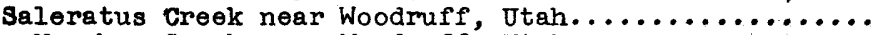

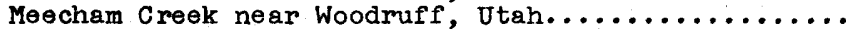

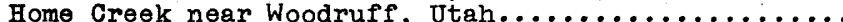

Woodruff Creok at Putnam Ranch near Woodruff, Utah.... South Fork Woodruff Creek near Woodruff, Utah.......

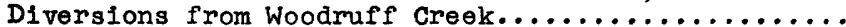

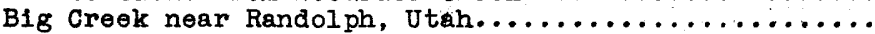

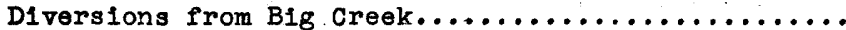

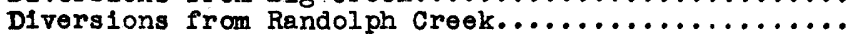

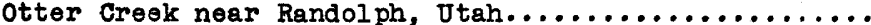

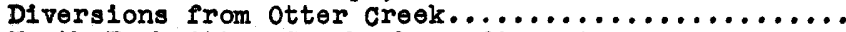
North Fork Otter Creek above diversions near

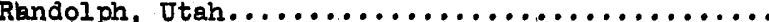

Middle Fork Otter Creok above diversions near

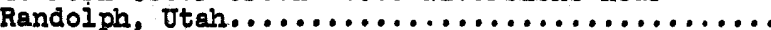

South Fork otter Creek above diversions near

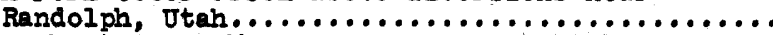

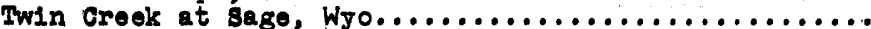

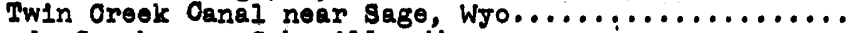

Leods Creok near Coker1lle, Wyo..................

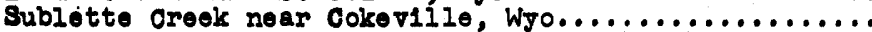

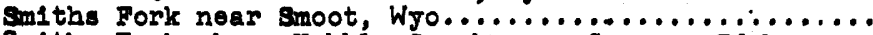
Sinlths Fork above Hobble Creok near Geneva, Idaho.....

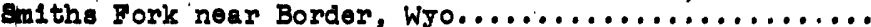
Sint ths Fork at Cokev1lie, Wyo..................

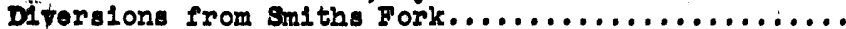
Hobble Creek near Geneva, Idaho................. Howlend Creok near Cokeville, Wyo..............

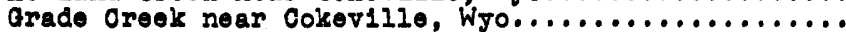
Pine Creok above diversions near Cokeville, Wyo.....

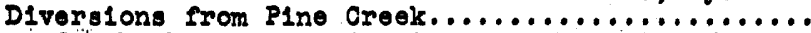
PIne Orbek above Covey Canal near Cokevilio, Wyo.... Spring Oreok above Covey Canal near Cokevilio, Wyo.. spring Creek to Collette Creek near Cokeville, Wyo..

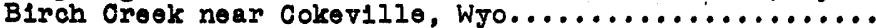
H10kman Canal near Cokevilie, Wyo................

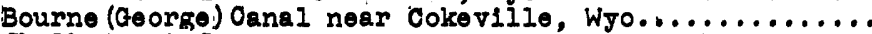
Chalk Creek Canal near Cokeville, Wro............... Thoma s Fork near Geneve, Idaho................. Thomes Fork above diversions noar Geneva, Idaho........

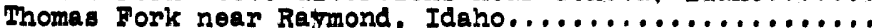
Thome Fork at mouth, near Border, Wyo............

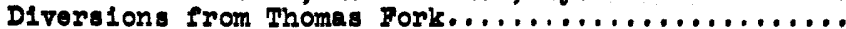

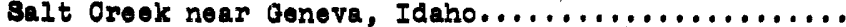
Pruess Oreok near Geneva, Idaho................

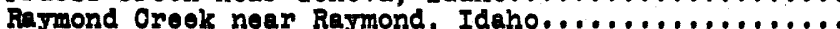
Montpel1er Oreok near Montpelier, Idaho............. Montpelier Oreok at 1rrigators welr, near Montpolier,

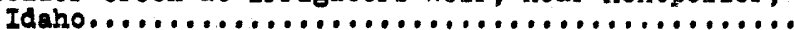

Montpelier Oreek bolow diversions at Montpolior, Idaho Diversions from Montpoller, Creok...............

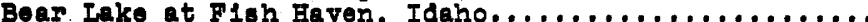

Bear Lake at Iffton near st. Oharles, Idaho.......... Bear Iake outlot canal near Parls, Idaho............

Oottonwood Oreek near Laketown, Jtah................

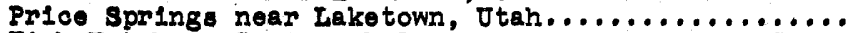
Flah Hatohery springs below Lower Round Vailey Canal

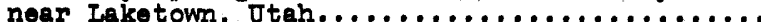

Upper Meadowv1lie Canal near Laketown, Utah.......
Drainage area square miles) 6,000

60.6
-

$1889-$

Perlod of record

$1942-48$.

$(1944,45)$ bc.

$(1946,47) \mathrm{bc}$.

(1945) bc.

80.5 1942-

- (1945) bc.

- $\quad(1944,45)$

- $\quad 1943-45$.

$\begin{array}{ll}- & (1946,47 \\ - & 1943-45\end{array}$

- $\quad(1944,45) b c$

$(1944,45)$ bg.

$(1944,45) \mathrm{bg}$.

$(1944,45) \mathrm{bg}$.

$(1944,45)$ bc

$(1944,45) \mathrm{bc}$.

1939-44; (1945) bc.

(1947) bg.

$(1944,45)$ bc; (1947) bg. $(1944,45)$ bc; (1947) bg. $1939-44$; (1945) b c .

(1944-45) bc.

(1947) b g.

(1947) bg.

$246 \quad(1947) \mathrm{bg}$.

246 1943-

- 1944,45) bo.

- (1944) bc.

- 1943

- $\quad(1944-46)$ bc.

165 1942-

275 1942-

- $(1944-48)$ bc.

$86.11943-46$.

- (1944-48) bo.

- $\quad(1944-48)$ bc.

- (1944-48) bc.

- (1944-48) bo.

- (1944-48) bc.

- $\quad(1944-48)$ bo.

- (1944-48) bc.

- (1844.45) bc.

- (1944-48) bc.

- (1944-48) bc.

- $(1944,45) \mathrm{bc}$.

45.3 1939-

- $(1944,45) \mathrm{bc}$ (1946) bg.

- $\quad(1944-47) \mathrm{bg}$.

- $(1944,45) \mathrm{bc}$.

$37.6 \quad 1939=$

- 1943-44.

- $\quad(1944,45) \mathrm{bg}$.

- 1939-44.

\section{$50.8 \quad 1942$ -}

- (1944-48) bo.

- $\quad(1944)$

- 1945-

- $1845-$

- (1944) bg; (1945) bo.

- (1944) bg; (1945) bo.

(1944) bg.

(1944) bg; (1945) bc.

b In Bear River Hydrometric Data Tri-state Invest1gations Report.

c Irrigation season records only.

g Miscellaneous discharge measurements.

$h$ In flles of Logan Project office, Goological survey. 
Great Salt Lake tributaries--Continued Bear River tributaries-c-Continued. Cottonwood Creek tributaries-Continued.

Fish Hatchery Springs tributaries--Continued.

Upper. Round Valley Canal near Laketown, Utah...... Lower Meadowvilie Canal near Laketown, Jtah........ Lower Round Valley Canal near Laketown, Utah......

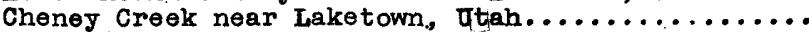

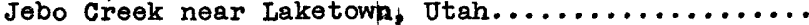

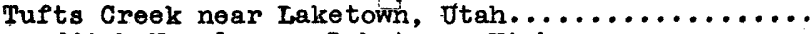

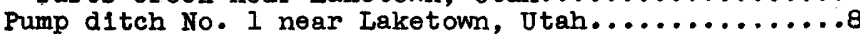

Laketown Creek near Laketown, Utah...............

Swan Creek below diversions near Garden Oity, Otah.... Diversions from Swan Creek:

Fish Haven Creek above diversions near Fish Haven,

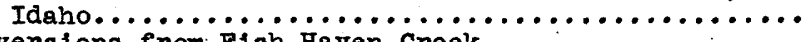

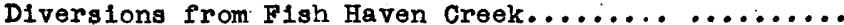

Fish Haven Creek below diversions at Fish Haven, Idaho

st. Charles Creok above diversions near St. Charles,

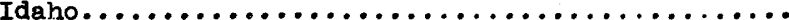

St. Charles Creek below diversions near st. Charles,

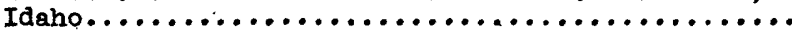

Ilttle Creek at st. Charles, Idaho..............

Diversions from st. Charles Creek.............. Spring Creek below spring Creek Canal near st.

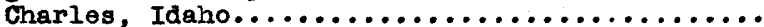
spring Creek Canal near st. Charles, Idaho......... Bloomington Creek near Bloomington, Idaho.......... Diversions from Bloomington Croek..............

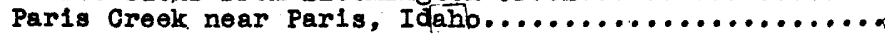

Parts Power Canal near Paris, Idaho..............

Diversions from Paris Creok.................. Parls Creok below diversions near parls, Idaho........ 811ght Canyon Creek near Paris, Idaho................ MIII Oreok above West Fork near Ifberty, Idaho........

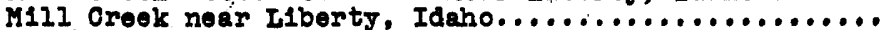
M111 Creok at Liberty BrIdge near Ilberty, Idaho...... Diverslons from M1Il oreok.................... North Oroek near I Iberty. Idaho.................. North Oreik bolow Bmigration Orook noar Liberty, Idaho.............................

North Oreek at Iiberty Bridge near Iiberty, Ideho...

DIVors Ion from North Oroek..................

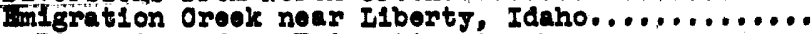
Diversions from Emigration ćreok...............

01d Bear Iajce outlet at Bern Road near Bern, Idaho.... Bonnington Oreok noar Bennington, Idaho............. Georgetown Oreok near Georgetown, Idaho............. Goorgotown Creek below diversions at Georgetown, Idaho Weat Fork near Georgetown, Idaho................

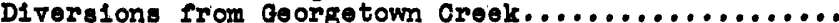

Stauffer Creok above diversions near Nounan, Idaho.... Stauffer Creek near Nounan, Idaho................

Stauffer Oreok at mouth near Georgetown, Idaho........

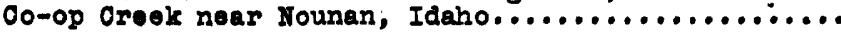
SkInner Orook at Nounan, Idaho..................

Pearl Oroek noar Goorgotown, Idaho.................

Bight Mile Oreok bolow diveralons noar soda springs,

Idaho................................

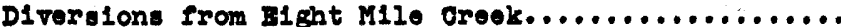

Balloy Greek befow diversions noar Soda springs, Idaho

Diversions from Balley Oreek...................

Diversion from Fomation Springs near Soda Springs,

Idaho. ..............................

Ledge Oreok near soda springs, Idaho................

Boda Orook at Iau BRhoh near sode springs, Idaho......

Sode Oreok near Sode springs, Idaho............... Soda Oroek below diverdions at soda springe, Idaho.... Diverslons from sode orook...................

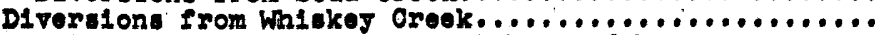
Trout Oreek at falls near Thatoher, Idaho...........

D1 versions from Trout oreek..................

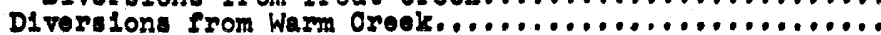

Drainage area

square miles)

Period of record

(1944) bg; (1945) bc.

(1944) bg; (1945) bc.

(1944) bg; (1945) bc.

$(1944,45) \mathrm{bg}$.

$(1944,45) \mathrm{bg}$.

$(1944,45) \mathrm{bg}$.

(1945) bg.

(1944) bg; (1945) bc.

$(1944,45)$ bc.

$(1944,45)$ bc.

$(1944,45) \mathrm{bc}$.

$(1944,45)$ bc.

$(1944,45)$ bc.

$(1944,45) \mathrm{bc}$.

$(1944,45)$ bc.

$(1944,45)$ bc.

(1944) bg; (1945) bc. (1944) bg; (1945) bc. 1943-47.

$22 \cdot 1$

$(1944,45)$ bc.

18.6 1943-47.

- 1943-47.

$(1944,45) \mathrm{bc}$.

$(1944-45) \mathrm{bc}$.

$1943-45$.

$1944-47$.

1943-47.

(1845) bo

$(1944,45)$ bo.

$1943-44$.

$26.5 \quad 1846-47$.

$-$

(1045) bo

(1944,45) bo.

$1943-44$.

(1944,45) bo.

(1944) bg; (1945) bo.

$(1944,45)$ bo.

22.2 i $1911-14,1939$.

$(1944-47)$ bo.

$(1944,45)$ bo.

$(1944,45)$ bo.

$(1944,45) \mathrm{bg}$.

$1939-44$.

$(1946,47)$ be.

$(1944,45)$ bo.

1939-44.

$(1944,45) \mathrm{bg}$.

(1944-47) bo.

$(1844,45) \mathrm{bc}$.

$(1945)$ bo.

$(1944,45)$ bo.

$(1944,45)$ bo.

$(1844,45)$ bo.

$1023-26$.

1913-26; 1928-29.

(1045-47) bo.

(1944,45) bo.

(1944) bg; (1845) bo.

(1945) bo.

$(1944,45)$ bo.

$(1944,45)$ bo.

b In Bear RIv0r Eydrometrio Data Tr1-8tate Invest1gations Report.

- Irrigation seas on reoords only.

8 M1scollaneous d1scharge measurepnents. 
Great Salt Lake tributaries--Continued. Bear River tributaries--Oontinued.

Williams Creek below diversions near Cleveland, Idaho.

Divers ions from Williams creok............... Cottonwood Creek near Swan Lake, Idaho.............. Cottonwood Creek near Cleveland, Idaho............

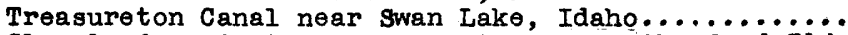
Cleveland Irrigation Company Canal near Gleveland, Idaho Mink Creek below Dry Fork, near Mink Creek, Idaho..... Mink Creek near Mink Creek, Idaho.................

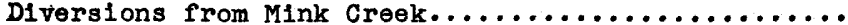
Twin I Iakes Canal near Mink Creek, Idaho........... Preston-RIverdale and Mink Creek Canal near Mink

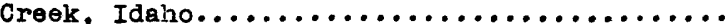

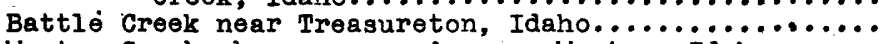
Weston Creek above reservolr near Weston, Idaho........ Weston Creek below reservoir near Weston, Idaho....... Weston Creek above McCaulic Canal near Weston, Idaho..

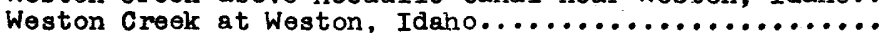

Diversions from Weston Creek.................

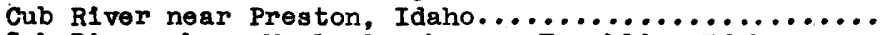
Cub River above Maple Creok near Franklin, Idaho......

Oub River at Frankiln, Idaho $\ldots \ldots \ldots \ldots \ldots \ldots \ldots \ldots \ldots$ Cub River-Worm Creek Canal at head near. Preston,

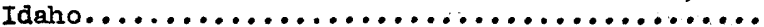
Cub River-Worm Creek Canal near Preston, Idaho...... Mapleton Canal near Preston, Idaho............... Preston-Whitney Canal near Preston, Idaho...........

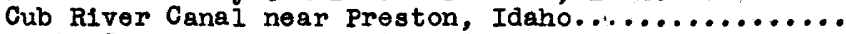

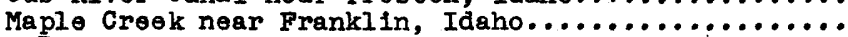

Diversions from Maple Creek.................. Maple Creek below diversions near Franklin, Idaho... Worm Oreek near Preston, Ideho................. HIgh Creek near Richmond, Utah................ D1 versions. from Elgh Crook.................. High Creok below diversions near Richmond, Jtah..... Oherry Orook (main channel) near Rlchmond, Otah..... Oherry Croek (BIrch Crook Ohannel) noar Ríchmond,

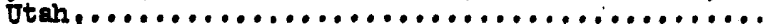

Diveralons from oherry oreok................. Oherry Oroak bolow ditersions near Rlchmond, Utah... Sumit oreok above diversion noar smithe101d, Otah... Biroh Creok at mouth near Smithrield, Jtah..........

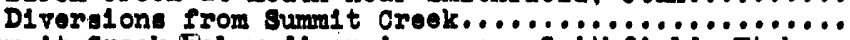
Sumit Oreok biolow divorsions noar smithelold, Jtah... L1ttlo Bear RIver noar Parad1se, Utah..............

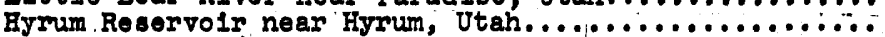

L1ttle Boar Rfver noar Hymum, Utah............... Fast Pork I1ttle Bear R1ver near Avon, Utah......... Pole Creek at mouth near Avon, Jtahr. ........... D1versions from Ilttio Bear River................ Logan River above State, Dam near Logan, Utah......... Logan River near Logan, Utah................... Logan River below State Dam near Logan, Utah........ Logan River below Logan Northern Canal near Logan,.

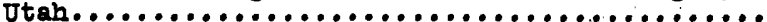

Utah Power \& Ilght Company's Tallrace near Logan,

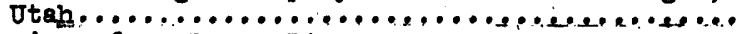

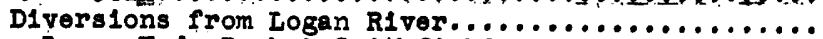
Logun, Bjde Park \& Smlthfleld Canal near Logan,

Utah...........................

Logan Northern Canal near Iogan, Utah.......... Blacksmith Fork at Hardware Ranch noar Hyrum,

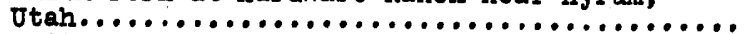

Blacksmith Fork at munlolpal power plant near Hyrum, $\tan \ldots \ldots \ldots \ldots \ldots \ldots \ldots \ldots \ldots \ldots \ldots \ldots \ldots$

Blackam1th Fork above Utah Power i ight Oompany's dam noar Hymum, Jtah...........

Blacksmlth Fork (above tallrace) at Utah Power \& Ilght Company's plant noar Eyrum, otah.......

Blacksmith Fork bol ow Utah Power \& II ght Company's plant near Hyrum, Utah............ reinage area squaro miles)

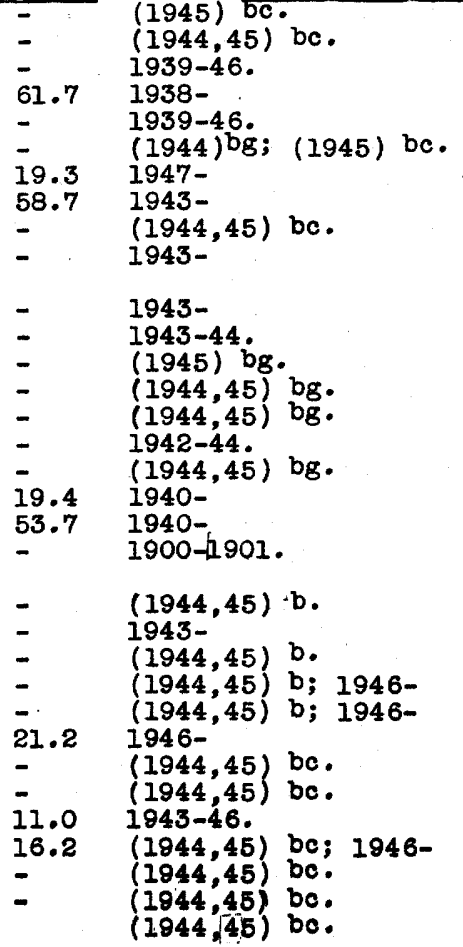

$=$

$(1944,45)$ bo.

$(1944,45)$ bo.

$(1944,45)$ bo.

1944,45 bo.

$(1944,45)$ bo.

$(1944,45)$ bo.

$(1944,45)$, bo.

20.3 1938-

220 1938-

$2221938-$

49.7 1827-30; 1938 -

$(1945-47) \mathrm{bg}$.

(1939-) \&1; $(1944,45) / \mathrm{Bo}$.

$218.1913-$

- 1896-1912.

- 1913-14.

- 1915-17.

- 1913-

-. $\quad(1944,45)^{b c}$.

- $\quad 1904+7$; 1909 -

- $\quad 1904-7 ; \quad 1909-16 ;(1944,45)$ bo.

150 1843-

1829-35.

260

1900-1908; 1913-

1814-16.

1900-1902; $1804-10 ; 1814-16$.

b In Bear R1ver Hydrometric Data Tri-state Investigations Report.

- Irrigation soas on records only.

8 Misollaneous discharge measurements.

1 In reports of Iittio Bear RIver Water Commissioner. 
Great salt Lake tributaries--Continued.

Bear River tributaries--Continued.

Littie Bear River tributaries--Continued.

Logan River tributaries--Continued.

Blacksmith Fork tributarles--Continued.

Hyrum City power canal (Blacksmith Fork power

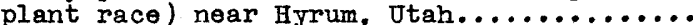

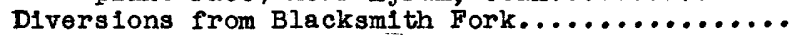

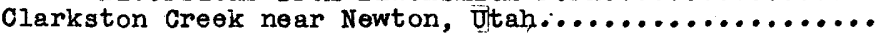

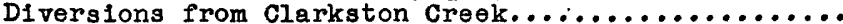

Malad River below springs, near Malad, Ijaho...........

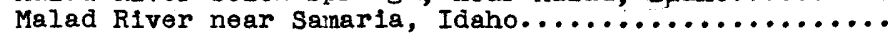

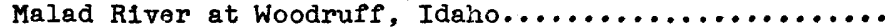

Warm Springs Canal near Samaria, Idaho............

Littlo Malad River above Elkhorn Reservoir, near

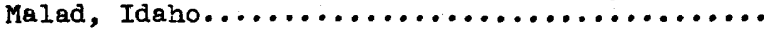

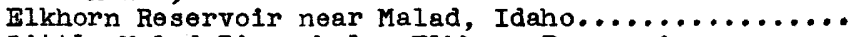

Little Malad River below Blkhorn Reservolr, near Malad, Idaho.......................

Little Malad River below Sand Ridge dam site, near

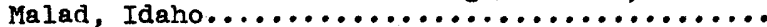

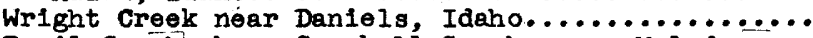

Devil Creek above Campbeli Creek, near Malad,

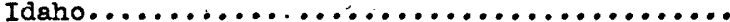

Devil Creek above Evans dividers, near Malad,

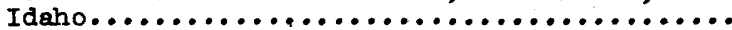

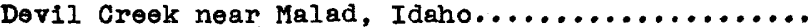

Deop Creek above Third Creek, near Malad, Idaho...

Deep Oreek below First Creek, near Malad, Idaho...

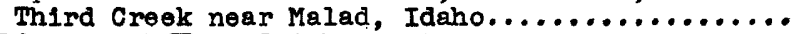

Box Elder Creek noar Brigham, Jtah...............

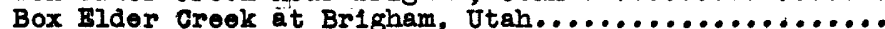

Weber River above Sith and Morehouse Creek near oakley,

Otan................................

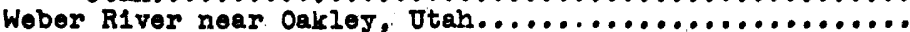

Wobor RIver noar Coalvilie, Jtah..................

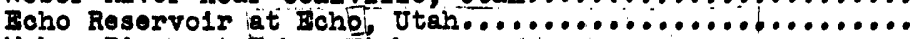

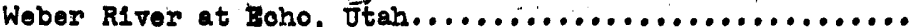

Wober R1ver at Dovils slide (oroydon), Jtah..............

Wobor RIver at Gatewry (noar Jinta), Utah..............

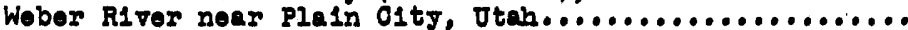

Smlth and Morehouse Oreel near Oakley, Utah..........

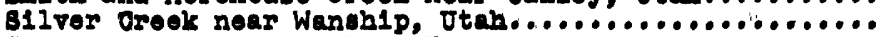

Ohalk Creak at Coelv1110, Jtah.....................

Lost Creek noar Croyden, Jtah....................

LQst Croek at Dovilo sIide (near arorden L Dtah.......

Bast Canyon Reservolr (on Bast Canjon Creok) near

Morgan, $\delta t a h . \ldots \ldots \ldots \ldots \ldots \ldots \ldots \ldots \ldots \ldots \ldots \ldots \ldots \ldots$

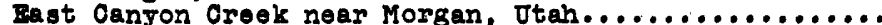

Hardscrabblo Creok near Porterv1110, Jtah............

South Fork Ogden River (head of Ogden River) near

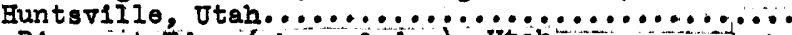

Ogden R1ver at Eden (above Ogden), Utah

Ogden R1ver near Ogden, otah....................

Ogden RIVer below PIne VIew/ Dam, near Ogden, Utah......

Ogden River at Powder Mili, near Ogden, Jtah..........

M1ddle Fork Ogden R1vor at Huntsville, Utah..........

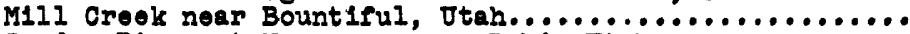
Jordan R1ver at Narrows, near Loh1, Dtah...............

Jordan R1ver at Balt Iake 01ty, Utah................

Jtah Lake near span1sh Eork, otah................

Utah Lake at Ceneve, near outlet, Dtah.............

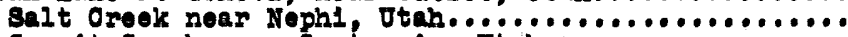

Surmit orook near Bantaquin, Jtah...............

Payson (Peteetnoot) Croek near Paycon, Dtah.........

Pajson (Poteotneot) Orieok ñear pajoon, totah..........

\begin{tabular}{|c|c|c|}
\hline $\begin{array}{l}- \\
\overline{-} \\
\overline{-} \\
\overline{-}\end{array}$ & $\begin{array}{l}1904-10 ; 1914-17 . \\
(1944,45) \mathrm{bc} . \\
1939-47 . \\
(1944,45) \mathrm{bg} . \\
1931-32 ; 1940- \\
1940-45 . \\
1938- \\
1940-45 .\end{array}$ & , \\
\hline$\overline{-}$ & $\begin{array}{l}1911-13 ; 1931-32 ; \\
1940-\end{array}$ & 1940- \\
\hline - & 1940- & \\
\hline - & $\begin{array}{l}1945- \\
1931-32 .\end{array}$ & \\
\hline - & 1938- & \\
\hline $\begin{array}{l}- \\
\overline{-} \\
-\end{array}$ & $\begin{array}{l}1940-43 ; 1946-= \\
1931-40 \\
1931-32 \\
1931- \\
1931-32 \\
1918-21 . \\
1909-12 .\end{array}$ & \\
\hline $\begin{array}{c}163 \\
438 \\
732 \\
732 \\
1,100 \\
1,610 \\
2,060 \\
- \\
85.8 \\
253 \\
135 \\
283\end{array}$ & 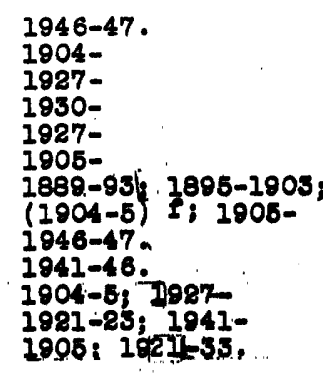 & ; $1919-$ \\
\hline $\begin{array}{r}144 \\
145 \\
24.9\end{array}$ & $\frac{1937-}{1941-}$ & \\
\hline $\begin{array}{c}148 \\
300 \\
310 \\
321 \\
- \\
= \\
2,960 \\
= \\
= \\
98 \\
27.5 \\
18.6 \\
28\end{array}$ & $\begin{array}{l}1921- \\
1895-96 . \\
1936- \\
1994-12 ; 1931-37 . \\
1937- \\
1889-90 ; 1897-99 . \\
1925-26 . \\
1913-14 . \\
1904 ; 1913- \\
1942 ; \\
1889-96 . \\
1896-1900 . \\
1925-38, \\
1905 ; 1010-16 . \\
1947-19 . \\
1910-16 .\end{array}$ & \\
\hline
\end{tabular}

New Frazmentary.

b In Bear R1ver Hydrometr10 Data Tri-state Invest1gations goport.

c Irrigation sergon reoords only.

1 In reports of State Inglneer of Jtah.

M Mroellaneous disoharge measurements.

$j$ In reports of Weber RIver Water Oommiseloner. 
Great Salt Lake tributaries--Continued. Jordan River tributaries--Continued. Utah Lake tributaries--Continued.

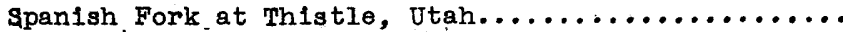

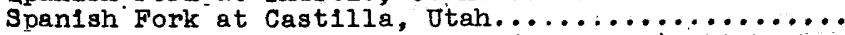

Span1sh Fork near Span1sh Fork (Mapleton), Utah.....

Span1sh Fork near (at) Lake Shore, Jtah...........

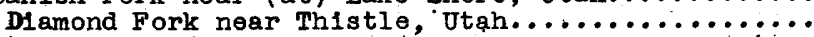
Strawberry tunnel at West Portal, near Thistle,

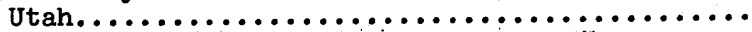

United states Bureau of Reclamation power canal near spanish Fork, Jtah................

Hobble Creek near Springville, Jtah.............

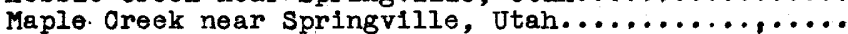

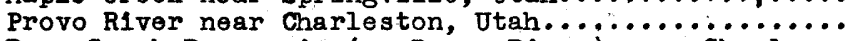

Deer Creek Reservolr (on Provo River) near Charles-

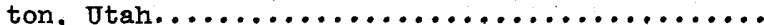

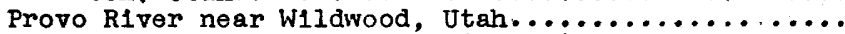

Provo River at Vivian Park, (Forks) Utah............

Provo River above Telluride Power Company's dam,

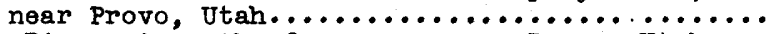

Provo River at mouth of canyon, near Provo, Utah....

Provo River at Denver \& Rio Grande Rallroad bridge,

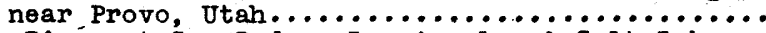

Provo Rivor at San Pedro, Los Angeles \& Salt Lake

Ral Iroad bridge, near Provo, Utah............

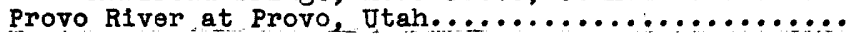
Weber-Provo diversion canal at oakley utah........ Weber-Provo diversion canal near Woodland, Utah... Round Valley Creek near Walisburg, Utah...........

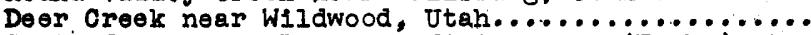
Amorican Fork above South Fork, near Amorlcan Fort,

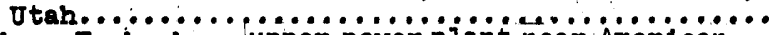
Amorloan Pork above upper power plant near American

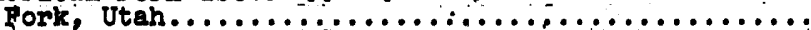

Amerloan Pork near Amerloan Fork, utah, ...........

South Fork American Fork near American Fork, Utah. Dry Crook now Alp1ne, Jtah..................

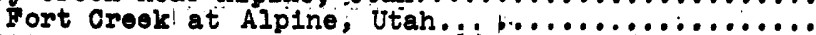
Ilttlo Oottonwood Oreok near salt Lake Oity, Otah... B1s Cottonwood Crook near salt Lake c1ty, Utah...... M1I Creok near salt Lake City, Jtah................ Surplus Canal at Salt Lake C1ty, Utah.............. Pariers Oreek near salt Lake clty, Jtah.............. Butgration Oreek near Salt Iake Clty, Utah.........

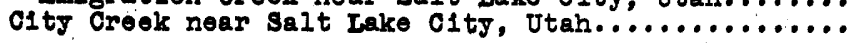

SEVIRR: IAKR BASIN

Mammoth Oreok (head of Sevier River) near Hatch, Utah.....

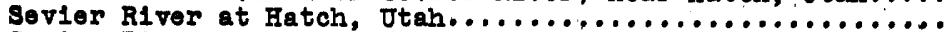

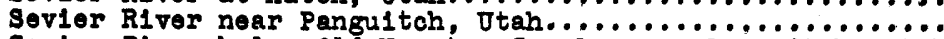
Sevier River bolow 0ld Houston Canal, near Panguitoh, Jtah

Sevier River near Olrcleville, Jtah.....................

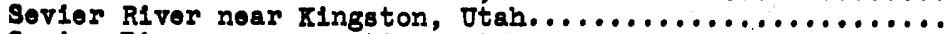

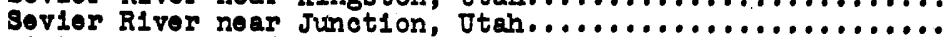
Plute Reservo1r (on Sevier Flver) near Marysvale, Dtah.... Sovior River bolow Plute Dam, noar Marysvale, Utab........ Sovler RIver at P1tts Ranch, noar Marysvale, Jtah..........

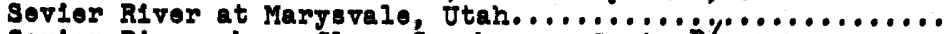

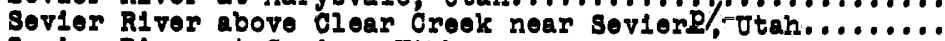

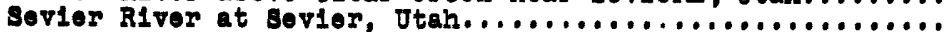

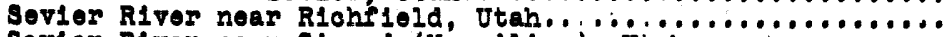

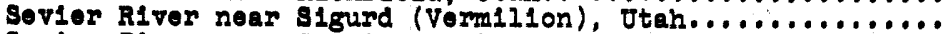

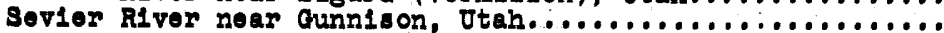
Soviar River bolow san P1toh R1ver, near Cunnison, Utah... Sev1er River at Olarko Bridgo, noar Payotto, Utah. .w...... Sov1er River at MoArt10's ford, near Fayette, Jtah......... Sovier Bridge Reservolr (on Sevier River) near Juab, Utah.

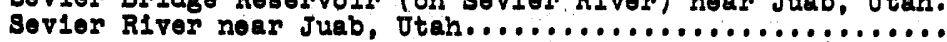
Snake Creok near Charleston, Jtah............. South Fork Provo RIver at Vivian Park (Forks), Dtah

Drainage area square miles)
490

670

670

700

155

-

105

$-$

$-$

600

$$
-
$$$$
67
$$

67

-

38.

71.9

26

30

43

55

$-$

21.3

50.1

29.0

19.2
Perlod of record

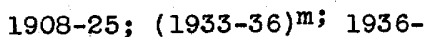
1919-25; (1933-36)m; 19361900-1901; 1903-17 n. $1903-7$; $1909-25$; $1938-$ $1907-17 ; 1940-$

1945-

1909-17.

1904-16; 1945-

1910-13.

1945-

1940-

1945-

$1911-$

1905-11.

1889-1906.

1905.

1903-4.

1933-34; 1937 -

1945-

$1931-$

1945-

1945-

1945-

1911-

1912-15.

$1945-$

1889-90; 1897; i800-1801;

$1912-15$.

$1047=$

1898-1813; (1914-30) 0.

1898-1913; (1914-30) б.

1898-1913; (1914-30) 0 .

$1942-$

(1814-30) 0 .

1898-1913; (1914-30) 0 .

1898-1913; (1914-30) 0 . m In Reports of Spanish Fork Water Conmission.

n Beginning 1811 add flow of Bureau of Reclamation Power canal to got flow of river.

- In Reports of City IngIneer, SaIt Lake Gity, Jtah.

p Published as Sevier River at Sevier, Jtah, 1911-16.

\section{1}

260

$-$

850

1,110

2.390

2,440

2,440

700

2,70

3.340

3,990

4,880

4,960

5,020

5,120

5,120
$1912-14$; $1915-19$.

1911-28; $1939-$

1914.

1812; 1914-27.

1914-

$1911-16$.

1914-

1911.

1906 6-11.

1912-14.

1911-16; $1939-$

1916-29.

$1916-18$.

1912; 1914-

1900-1917.

1917-

$1914-16$.

1914 ; 1915.

1914- 
Sevier Lake Basin--Continued.

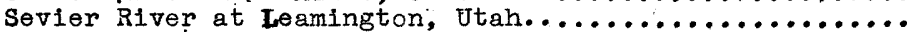

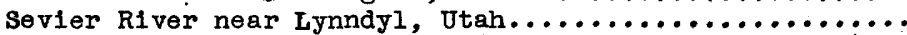
Delta. and Melville Reservolr (offstream Sevier River)

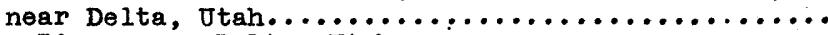

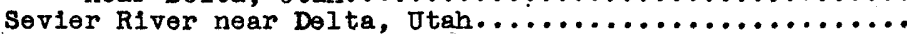
Gunnison Bend Reservoir (offstream Sevier River) near

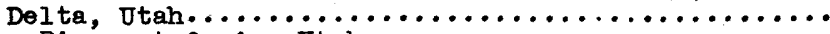

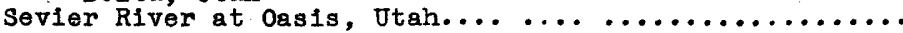

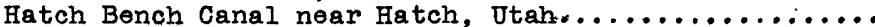

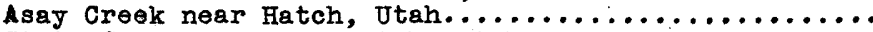

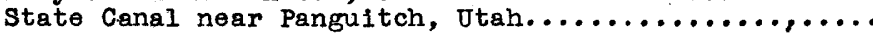

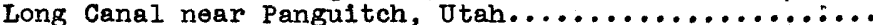

East Panguitch Canal noar Panguitch, Utah........... Panguitch Creek above canals near Panguitch, Utah..... Panguitch Creek below canals at Panguitch, Jtah........ Barton and Le Fevre Canal near Panguitch, Utah........

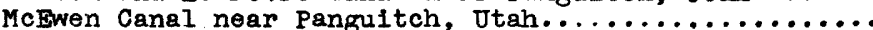

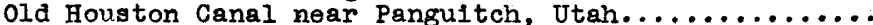

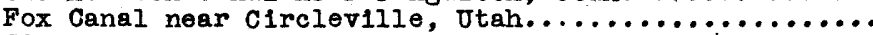
Circleville Canal near Clrcleville, Utah..............

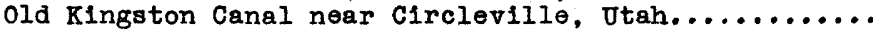

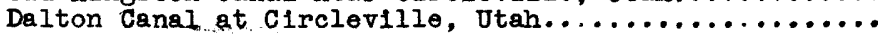
Mitchell slough:

Mitchell slough Canal near Junction, Utah..........

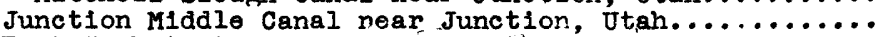

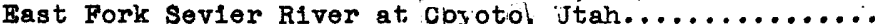
East Fork Sevier River above Otter Creek, near Coyoto,

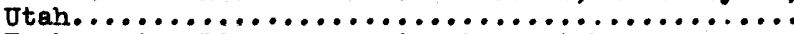

Bast Fork Sevier River near Kingston, Utah............. East Fork Sevier R1ver near Junction, Utah............

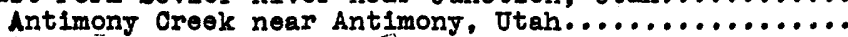

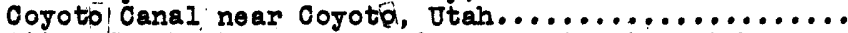
otter Creek above reservolr, near Coyoto, Jtah...... Otter Crook Reservo1r (on Otter Creek, offstream Gast Fork Bevier River), near Antimony (Oojoto) Otan: ..............................

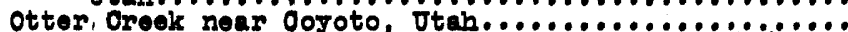

Otter Oreok Reservolr foeder canal at hoad; near

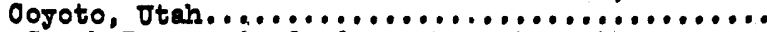
Otter Orook Reservolr fooder canal at mouth, near Ooyoto, Jtah.........................

Kingston Oaral at zingston, otah a. $\ldots \ldots \ldots . . \ldots \ldots \ldots$ PIne (Bullion) Oreak (upper tation) ñotir Maryavalo, tah...............................

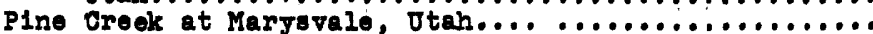

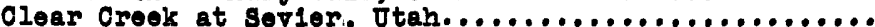

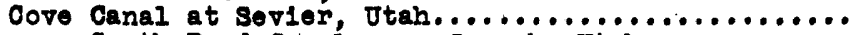

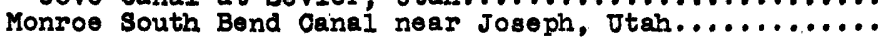

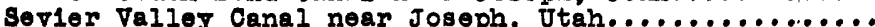
Sevier Valley Canal at Flsinore, Dtah...............

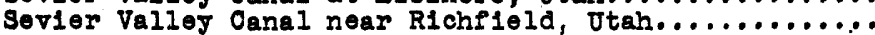

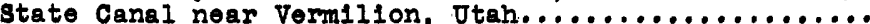
State Canal near Aurora, otah....................

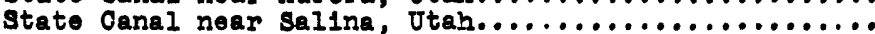

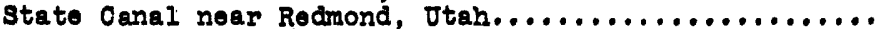

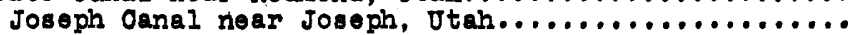

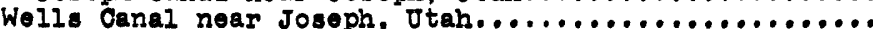

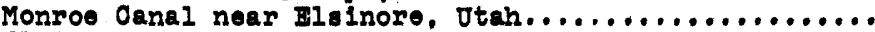

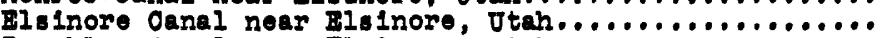

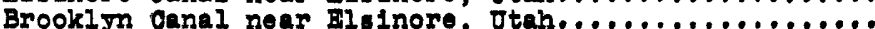

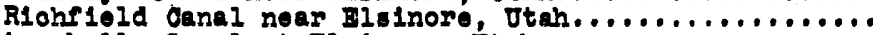

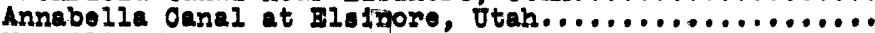

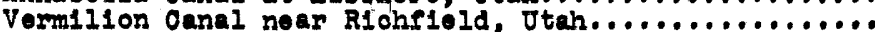
Rookyford Canal noar Vormilion, Jtah.............. Sal Ina Grook near Salina, Utah..................

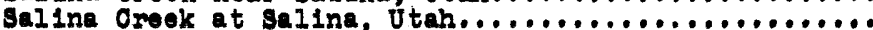
West Vlow Danal at Redmond, Utah.................

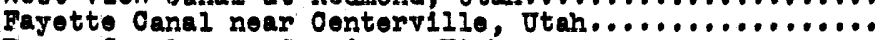

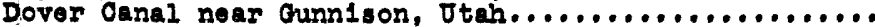

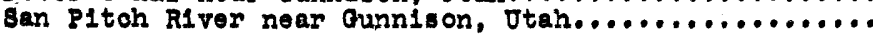

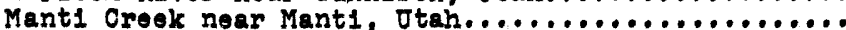

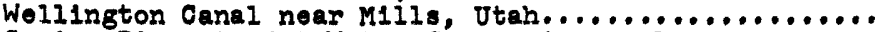
Sevier River Land de Water Company's canal near

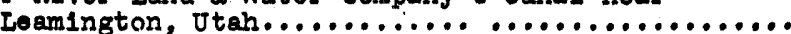

Sovier RIver Land \& Water Company's canal above Fool

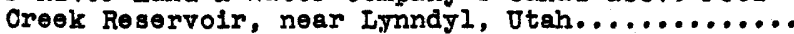

Drainage area
square miles)

5,800
6,270

$1914-18$

$1889-93 ; 1912-14$

$7,380 \quad 1914-17$.

$8,080 \quad 1914-19$

1914 ; $1916-19$.

$1912-14$

1913-19.

$1914-19$.

$1914-19$.

1915-20.

$1915 ; 1917-18$.

1915-19.

1914-19.

$1915-19$.

1914-19.

1914-19.

$1914-19$.

1914-19.

$1914-19$.

1915-19.

1914-19.

$\begin{array}{ll}1.260 & 1915-16 . \\ - & 1913- \\ - & 1912-13 . \\ - & 1947-48 . \\ - & 1916-19 . \\ & 1915-20 .\end{array}$

- $\quad 1914811947-$

- 1913-18.

- 1914-18.

- 1816-20.

- 1914 -19.

- 1918-18.

$169 \quad 1914 ; 1918-19$.

160 1914-19.

1914-19.

1912-19.

1913.

1912-19.

1813.

1913.

1913.

1913-19.

1914-19.

1014-19.

$1914-19$.

1814-19.

1914-19.

1914-19.

$1914-19$.

$1914-19$.

1914-35.

1900-1901.

$1914-19$; 1942 -

1914-19.

$\begin{array}{ll}- & 1914-18 . \\ 886 & 1914-18 . \\ & 1900-1905\end{array}$

$\begin{array}{ll}- & 1914-18 . \\ 886 & 1914-19 . \\ & 1900-1905\end{array}$

$886 \quad 1900$.

1914-18.

- 1914-18.

- 1914. 
Sevier Lake Basin--Continued.

Drainage area

Sevier River tributaries--Continued.

(square miles)

Perlod of Record

Sevier River Land \& Water Oompany's by-pass near

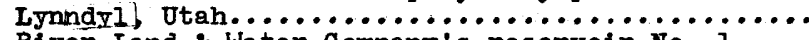

Sevier River Land \& Water Company's reservoir No. i

(Fool Creek Reservoir) near. Lynhdyl], Utah.........

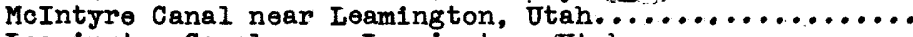

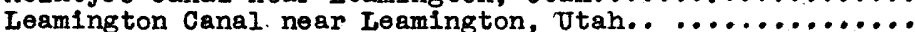

Canal A (Delta and Mellville Canal) near Delta, Utah....

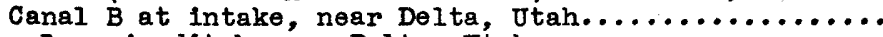

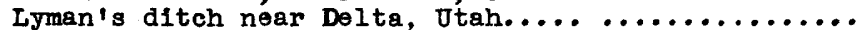

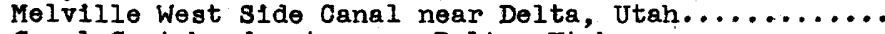

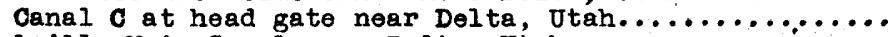

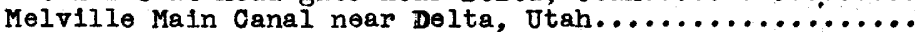

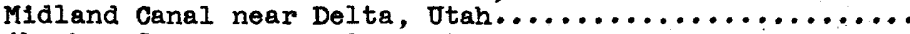

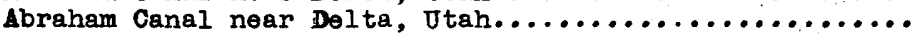

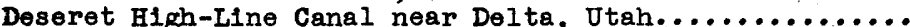

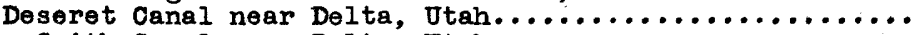

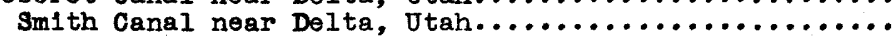

PAVANT VALLEY

Chalk Creek near Fillmore, Jtahtel..................

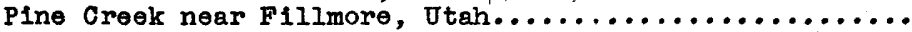

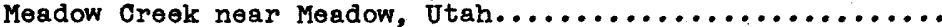

Corn Creek near Kanosh, Jtah.....................

BEAVER RIVER BASIN

Three Creek (head of Beaver River) near Beaver, Utah.....

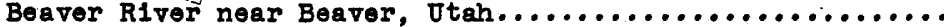

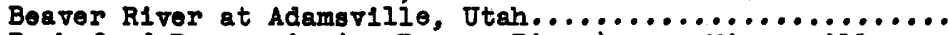
Rockyford Reservolr (on Beaver River) near Minersvilio,

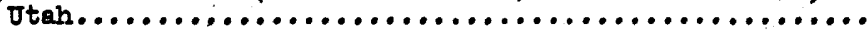

Beaver Rivor at Rockyford Dam, noar Minersvilie, Jtah.....

Beaver River at Minergville, Jtah.....................

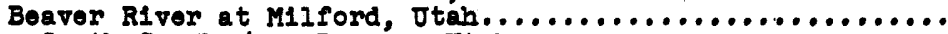
South Oreok near Beaver, Jtah....... Morth Fork North Oreek (head of North Oreok) near Boaver

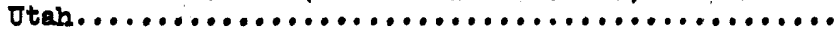

North Fork North Creek near Beaver, Jtah............. South Porl North Croek near Beavor, Jtah............

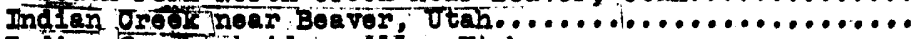
Indian orodr at Adamsvilí, 0 tah.................. MInersvillo Uanal at MInersvilio, Utah................

PAROWAN VALIEYY

Center Oreek near Parowan, Jtah....................

CBDAR OITY VALIEIY

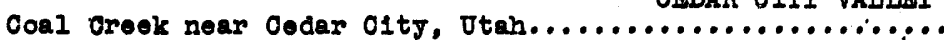

MINOR BASINS IN NEVADA

Thousand i Springs oreok noar Tacoma, Nevada............ Overland oreok near Ruby valley, Hovade................

Bnake Oreek near Baker, Nevada

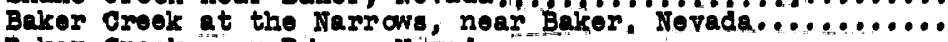

Baker Groek noar Boker, Névada

Iohman Oreok noar Baker, Novada.....................

Cloveland Oreok noar 0ocoola, Nevada..................

Currant oreok at ranger station noar ourrant, iovada.......

Currant Oreok near Currant, Nevada....................

B1g Warm Spring noar Duckwater, Novada.................

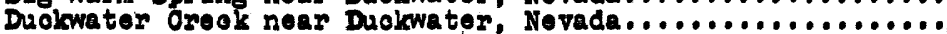

Lees Orsek near Pahmump. Nevada......................

Intermittent springs near Pahrump, Nevada...............

Blroh oreok noar Austin, yevade.....................

\section{BAITON SMA BASIN}

Salton sea, Oal1f..............................

Alamo River near Brawley, dalif...................

How R1vor near BrawleJ, Cellf....................

$\begin{array}{ll}- & 1914 . \\ - & 1914 . \\ - & 1914-18 . \\ - & 1914-19 . \\ - & 1912-19 . \\ - & 1912 . \\ - & 1912 . \\ - & 1912 . \\ - & 1912 . \\ - & 1912 . \\ - & 1914 . \\ - & 1913-14 . \\ - & 1913-14 \\ - & 1913 ; 1914 . \\ & 1914 .\end{array}$

1914 ; $1943-$

1914 .

1914.

1914.

$\begin{array}{ll}19.2 & 1947- \\ 82 & 1906 ; 1914- \\ 272 & 1913-36 ; 1937- \\ 510 & 1937- \\ 512 & 1913- \\ - & 1908-13 . \\ - & 1914 . \\ & 1906 . \\ 6.9 & 1947- \\ - & 1906 . \\ 19.4 & 1906 . \\ 180 & 1947- \\ - & 1914-20 \\ & 1906 ; 1914 .\end{array}$

60

1942-

82

1915-19; 1935-

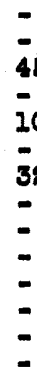

1810-13.

$1817-18$.

45 1813-17.

- 1847.

10 1913-15.

- 1847-

32 1914-16.

- 1913.

1913-17; 1923.

1815-16.

1815-17.

1816.

1916.

$1013-14$. 


\begin{tabular}{|c|c|c|}
\hline \multirow{2}{*}{\multicolumn{3}{|c|}{$\begin{array}{l}\text { Salton Sea tributaries--Continued. } \\
\text { Whitewater River-Basin: }\end{array}$}} \\
\hline & & \\
\hline $\begin{array}{l}\text { Snow Creok near Whitewater, Calif......................... } \\
\text { Southern Pacific Company's ditch near Whitewater, }\end{array}$ & - & $1921-31$ \\
\hline 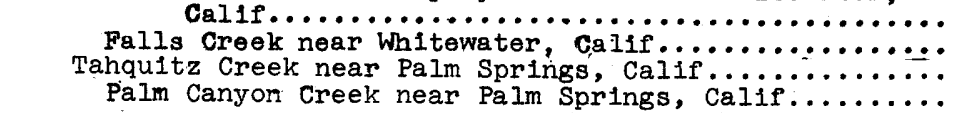 & 94.0 & $\begin{array}{l}1921-27 ; 1929-30 ; 193 \\
1922-31 . \\
1948- \\
1930-42 ; 1948-\end{array}$ \\
\hline
\end{tabular}

MOJAVE RIVER BASIN

Deep Creek (head of Mojave River) near Hesperia, Cal1f.... Mo.jave River at and near Victorvilie, Calif............. Mojave River at lower narrows, near victorville, Calif....

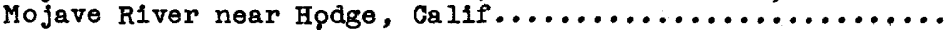

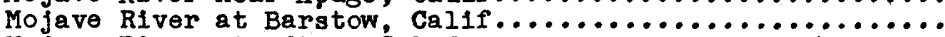

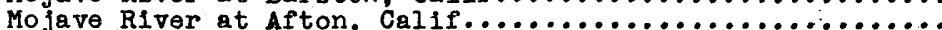
West Fork Mojave River near Hesperia, Calif.............

ANTELOPE VALIEX

Rock Creok near valyermo, Cal1f..................... Iittlo Rock Oreok near Iittle Rock, calif................

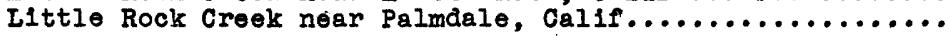

OWENS LAKR BASII

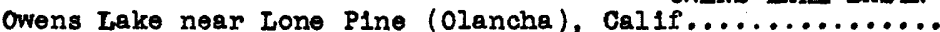
Owens River near Round valley, Calif................. Owens River at Pleasant Valley, near Blshop, Calif...... Owens River near Blg PIne (TInemaha), Galif............

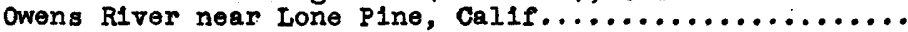

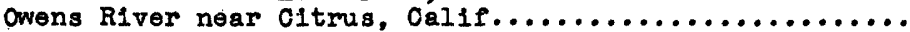
Rock Creok at Sherwin H1ll, near Bishop, Calif........ Rock Creek near Round Valley, Callf. o. PIne Creek at division box, near Blshop, Calif...... PIne Cresk near Round valley, Calif............... Owens River Canal near Bishop, Callf...............

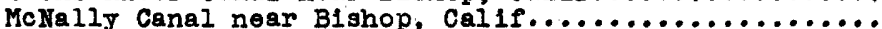

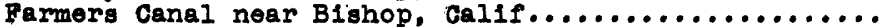
Bl shop Oreok near Blshop, Cal1f..................

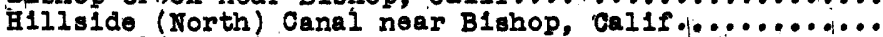
H1Ils1de J(South) Canal near Blshop; dal1f............ Powers Canal noar Blshop, oal1f...................

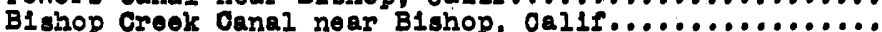
Collins (Goorge) Canal noar Bl shop, Cal1r............ Collins $(1.0$.$) Canal near Blshop, dallf..............$

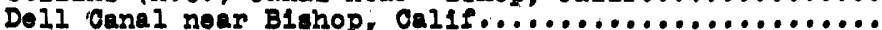
B1g PIne and Owens Rivor. Oanal near B1shop, Calif dot;:; Rawson Canal noar Blshop, Cal1f................... sanger Canal at Alrord oallf..................... Baker Creek near B1s Pine, Cal1f................. B1g P1ne Creok noar B1g Pine, Calif............... TInemaha Oreok near BIs PIne (TInemaha), Calif........ Blich Oreok near B1g Plne (Tinomaha), Callf........... Taboose Croek near Abordeon (Tibbetts), Oalif.........

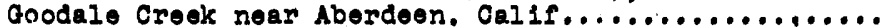
Division Creok near Indeperidence, oailf.............. Blghtmile (Sawmili) Orook near Indopendence, Calif..... Thlbault Creok near Independence, cal1f............. Bast side Canal near C1trus, Calif.................

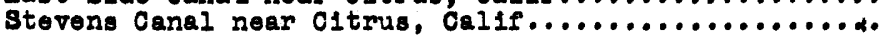
Oak Creok near Independence, Cal1f................. Ilttie PIne (Independence) Creok near Independence,

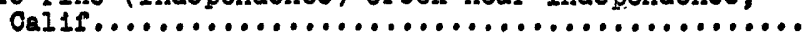

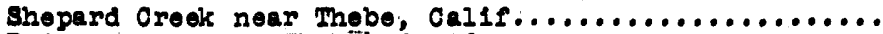
Balrs Crook near Thobleb cal1f...................

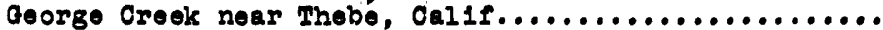

Lone Pine Oreok near Lone Plne, Cal1f............... Tuttle Groek near Lone PIne, Cal1f............... Cottonwood Oreek near Olanche, Cal1f................. Ash Orook near Ione Plne (Olanoha), Oal1f.............

$\begin{array}{cl}137 & 1929- \\ \overline{-} & 1899-1906 ; 1930-36 . \\ 530 & 1936- \\ - & 1930-32 \\ \overline{7} & 1930- \\ \overline{74.8} & 1929-32 \\ & 1930-\end{array}$

$23.0 \quad 1923-37 ; 1938-$

49.0 1930-

- $1896-99$.

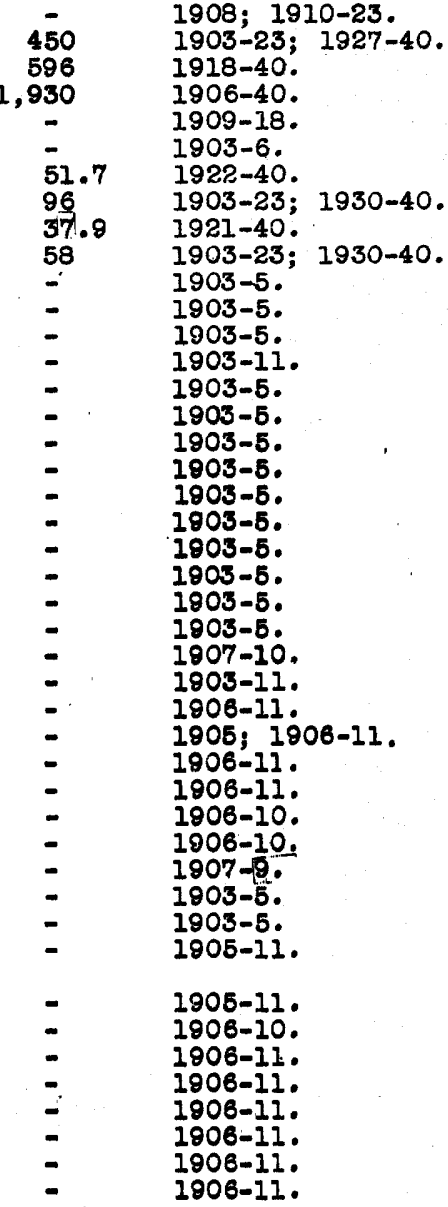


WALKER IAKE BASIY Drainage area
(square miles)

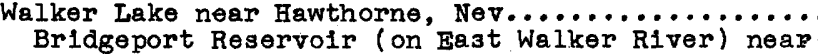

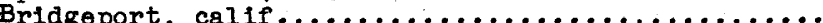

East Walker River (head of Walker River) near Brldgeport

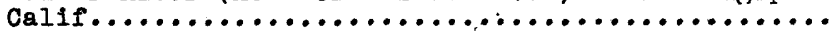

East Walker River above strosnider ditch, near Mason,

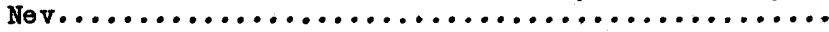
East Walker River above Mason Valley, near Mason, Nev...

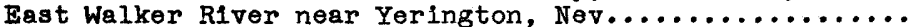

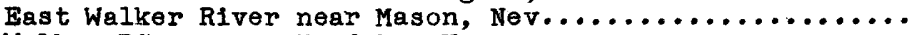

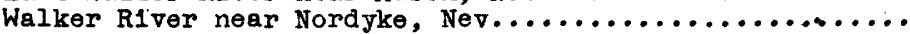

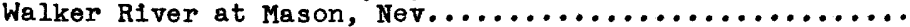

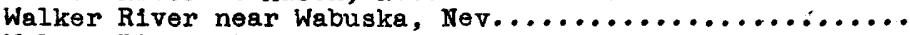

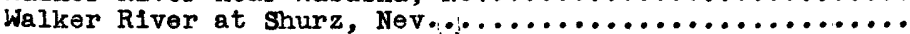
Roblason Creek near Bridgeport. Calif............. Buckeje Creek near Brilageport, Calif.................

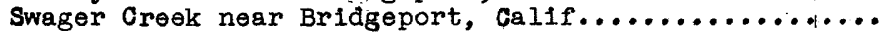
West Walker River below East Fork, near Colevilie, Galif West Walker Rlver near Colevilie, Calif.............. West Walker River at Hoye Bridge, near Wellington, Nev West Walker River near Wellington, Nev.............

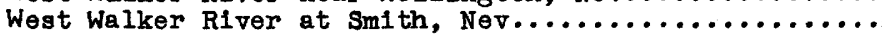

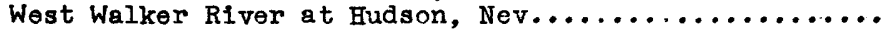

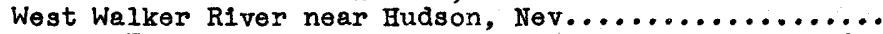
East Fork West Walker RIver near Bridgeport, Cailf..

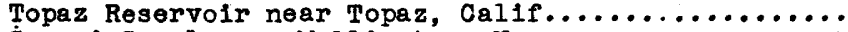

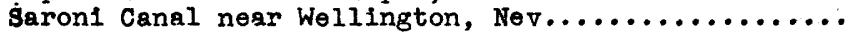

\section{HUMBOIDT-CARSON SINK BASIN}

Carson River Basin:

Carson River, Bast Fork (head of Carson River), above Soda springs ranger station, near Markleeville,

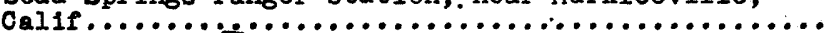
Carson River, Bast Fork, at silver King Valley, near

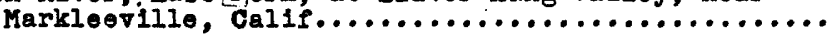
Carson River, Fast Fork, near Markleeville, Calif....... Carson R1ver, Fast Fork, at Cal 1fornia-Nevada Stato I1no Carson River, Bast Fork; noar Gardnorvilie: (at Rodenbah's ranch and Horseshoe Bend), Nev.

Carson River near Garson C1ty, Nev..................

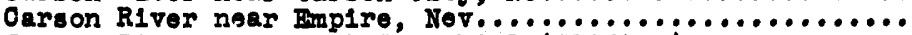
Carson R1ver noar Fort Church1li (Ol1fton), Nov......... S1lver King Greok near Ooleville, Cal1f............. Wolf Oreok near Markloeville, Oailf................ silver Groek below Pennsylvania Oreok, near Markioe-

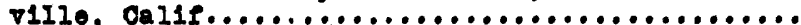

silver Creek near Markloovilio, Oelif............... Markleoville Croek above Grover Hot Springs, near

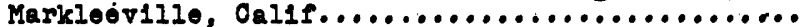

Markleeville Creok above Markleoville, Gallf......... Markloeville Crook at Markleov1lle, cál1f............. Ploasant Valley Creok above Baymond Canjon Creok, near Markleev1lie, Cal1f................... Ploasant Vallor Crook near Markleovilie, Cailf......

Wost Fork Carson River abovo Woodfords, Cél1f........

West Fork Carson RIver at Woodfords, Cal1f.r. .........

Clear Oreek near Carson O1ty, Nev.................. Humboldt River Basin:

Bumboldt River noar Blko, Nev.................... Eumboldt River near Oarlin, Nov................... Humboldt River at Pallsade, Nov...................

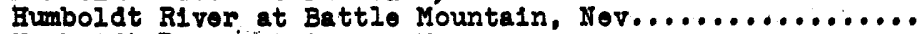
Humboldt R1 ver at. Comas, Nev....................... Humboldt River near Golconda, Nev...................

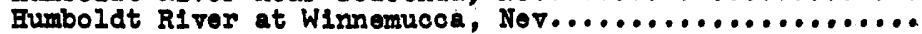
Humboldt RIver near Rose Oroek, Nev.................. Humboldt Rivor noar Imlay, Nov.................... Eumboldt River near tumboldt, Nov................... Ry Patoh Reservolr noar Ryo Patch, Nov.............. Fumboldt R1ver near Rye Petoh, Nev.................. Humboldt River near oreana Nev....................

$\begin{array}{cl}- & 1928- \\ 362 & 1931- \\ 362 & 1911-14 ; 1921- \\ - & 1947- \\ - & 1916-18 ; 1921-24 \\ - & 1902-8 . \\ 1,230 & 1910-16 . \\ - & 1895 . \\ - & 1910-16 ; 1921-23 \\ - & 1902-8 ; 1920-34 \\ 2850 & 1913-33 . \\ - & 1910-14 . \\ - & 1910-14 . \\ - & 1911-15 . \\ 182 & 1938- \\ 504 & 1902-10 ; 1915-38 \\ 521 & 1910 ; 1924-32 . \\ - & 1917-24 \\ 953 & 1914-21 . \\ - & 1921-25 ; 1947- \\ 63 & 1910 ; 1944- \\ - & 1931- \\ - & 1920-23 .\end{array}$

At For seshoo Bend.

$r$ Reoords 1890-98; $1807-20$ at bridge and below 3 canals - not oquivalent. 
Humboldt River Basin--Continued.

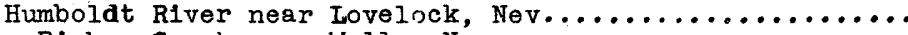

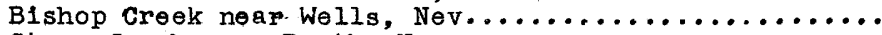

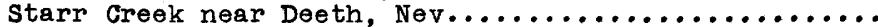
Marys River at Marys River cabin, near Deeth, Nev..... Marys River at Buena Vista Ranch, near Deeth, Nev.....

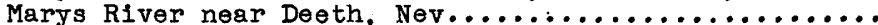
Marys River below Hot Springs Creek, near Deeth, Nev..

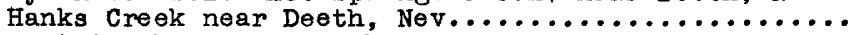

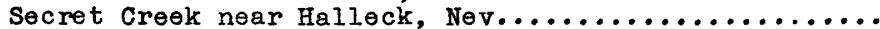

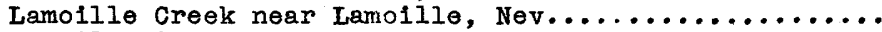

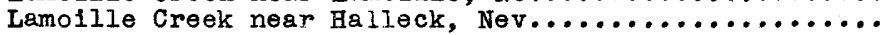
North Fork Humbolat River at Devils fete, near Halleck

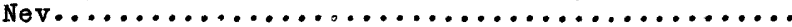
North Fork Humboldt River near Halleck (Peko), Nev.... South Fork Humboldt River near Lee, Nev............ South Fork Humboldt River near Elko, Nev...........

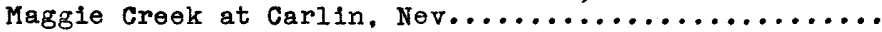

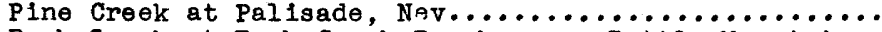
Rock Creek at Rock Creek Ranch, near Battle Mountain,

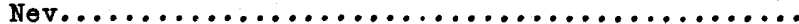

Rock Creek near Battle Mountain, Nev..............

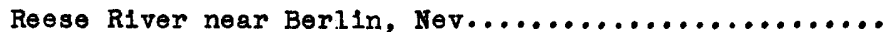

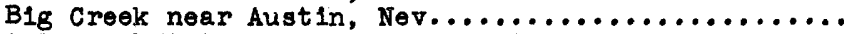
Littio Humboldt River at Chimney dam site, near

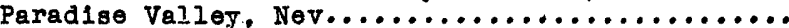

Little Humboldt River noar Paradise Valley, Nev........ Martiñ Creok near Paradise Vallev, Nev.............. Cottonwood Creek near Paradise Valley, Nev........ Cottonwood Creek at Paradise valley, Nev.......... Humboldt-Iovelock Irrigation, Iight \& Power Company's

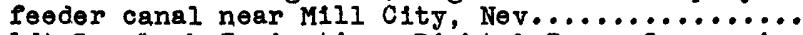

Humboldt-Lovelock Irrikation, Light \& Power Company's

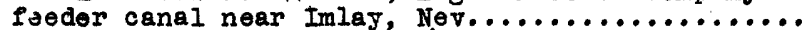

Humboldt-Iovelock Irrigation, Iight \& Power Company's

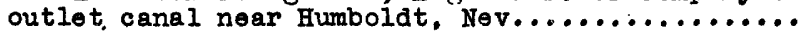

PYRAMID AND WINNEMICCA LAKRЯ BASIN

Pyramid Lake (at Pyramid Lake Sanatorium) near N1xon, Nev.

Lake Tahoo at Tahoe, Calif .....................

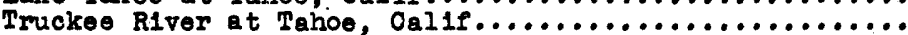

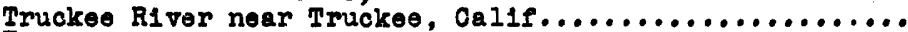

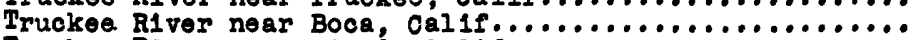

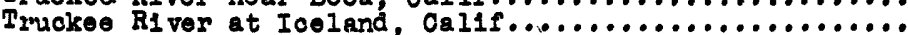

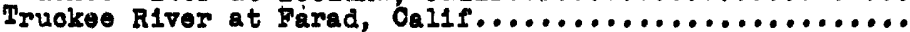
Truoleo River at Nevada-California state Iine........... Truckee River at Laughton, Nev.......................

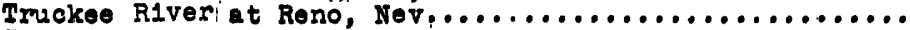

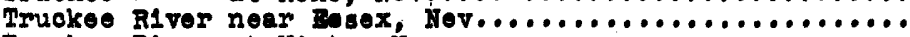

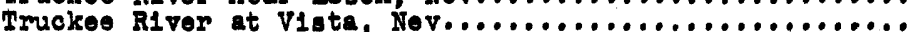
Truckee River at Olarks, Nev...................... Trucke RIver at Derby Dam, Nev....................

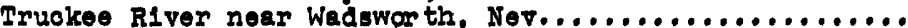
Donner Creek at Donner Lake, near Truckee, Cal1f......

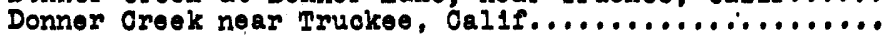
Prosaer Oreok near Fobart M111s (Truckeo), Cal1f...... Prosser Creok near Boca, dal1f.................. South Fork Prosser Croek near Tmickee, Oalif........

Ittle Truckeo River near Truokeo, Cal1f.............

Ittle Truoke River near Hobart Mills, Cal1f.........

Iittio Trucke River at Pine station and starr, Cailr.

Iittle Truckee River at Boca, Cal1f................ Webber crook noar Truckee, cal1f................ Independence Cresk below Independence Lake, Calif... Independence Croek noar Truckeo, Cal1f............. Franktown Creek (hëadwaters of calena Creek) at

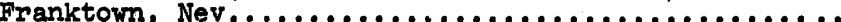

arlena Creek (headwaters of Steamboat Creek) near

Washoe, Nev............................

Steamboat Creek at Steamboat Springs, Nev............

Lake Winnemucca inlet near Wadsworth, Nev,...........
Drainage area square miles) 14,200 $-$ $3 \overline{55}$

415

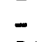

25

245

830

1,020

1,150

$-$

-

$\overline{-}$

94

9

1,030

172

62

$-$

-

$-$

$-$

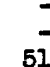

519

548

937

940

955

1.070

$-$

1,740

-

30

30
48

5.8

33.2

-

14

8.

- 1948 -

- 1913-14.

- 1900-1901.

- 1902-5.
Period of Record

1910.

1913-24.

1913-14.

1913-14.

1902-3; 1912-28.

$1943-$

1913-14.

$1917-24$.

1915-23; $1943-$

1913-19.

$1913-21$; $1943-$

1898-1900; $1902-13$.

$1945-$

1896-1932 ; 1936-

1913-24.

$1902-4$; $1912-14$; $1946-$

1915-17.

1896; 1918-25; 1927-29;

1946-

$13-16$

$1913-14$; 1916.

$1941-$

$1921-28$; $1943-$

1921-

1925-34.

$1944-$

1914-31; 1937-38.

$1946-$

1914-20; 19:21-41.

$1926-$

1900-1943

$1895-96$; $1900-1943$.

$1944-$

1890.

1912-37.

$1938-43$.

1898-1912.

1890 .

$1906-19$; 1947-

1889 .

1899-1807.

1907-15.

1907-10.

1902-5.

1909-10.

$1902-15 ;$; $1928-43$

3-4: $1907-12$.

$-10$.

1903-10.

1890; $1911-15$.

1909-10.

1902-7.

$1909-10$. 


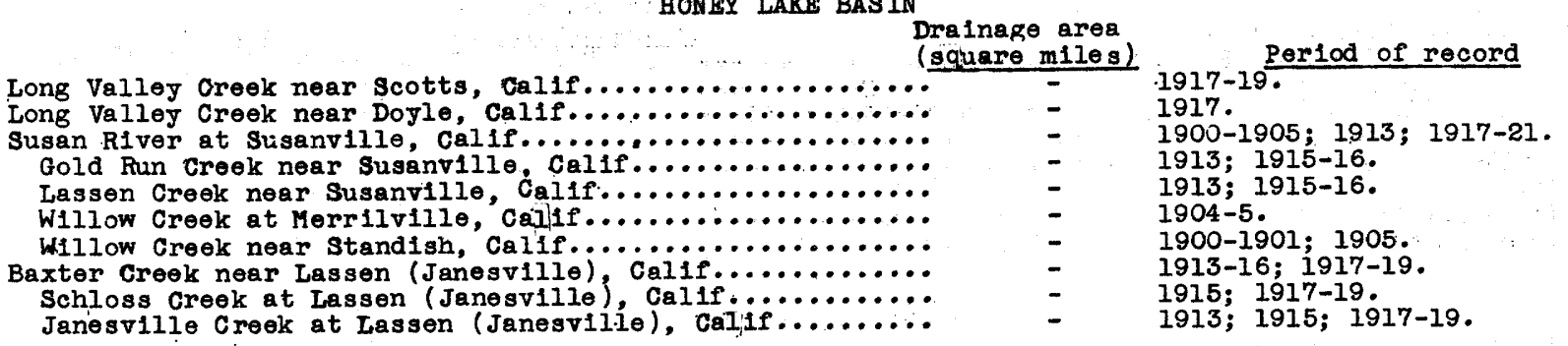

RED ROCK CREEK BASIN

Red Rock Creek near Red Rock, Calif..................

1917.

SURPRISE VALIEY DRAINAGE BASIN

Bidwell creek near Fort Bidwell, Calif................. Bldwell Creek at. Fort Bldwell, Callf................. Box Canyon Creek near Fort Bidwell, Callf..............

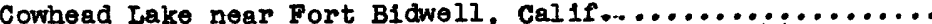
Twentymile Creok near Adel (Warner Lake), Oreg...........

Keeno Creek near Fort Bidwell, Cal if................. Fifteenmile Creek near Warner Lake, Oreg.............. Fifteenmile Creek below Rock Creek, near Fort Bidwell,

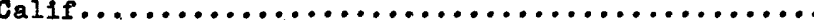

Twel vemile: Creok near Fort Bldwell, Calif........... Bàst Fork Horse Creek near Fort Bidwel1, Callif...... West Fork Horse Creak near Fort Bldwell, Calif......

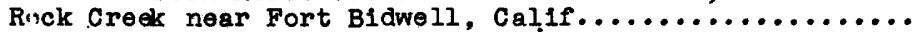
Deep Creek above Dismal Creok near Warner Lake, oreg....... Deep Creek below Dismal Creek near Warner Lake, Oreg....... Deep Creek at Big Valley, near Lakevlew, Oreg............ Deep Creek above Adel, oreg......................... Deep Creek at Adel, Oreg...........................

Dismal Creok above B1g Valley near Warner Lake, oreg....

D1 smal Creok nesr Warner Lake, Orelg..................

Camas Oreok near Plush, oreg.......................

Camas Creek below BIue Oreek, near Lakev1ew, Oreg....... Mud Greok near Plush ones......................... Orane Creok near Lakeviow, Oreg................. Drake Oreok near Adel, oreg....................... M. C. G1van ditoh near Adel, Oreg.................. Company ditoh near Adel, oreg.................... M. O. ditoh at Adel, Oreg.......................

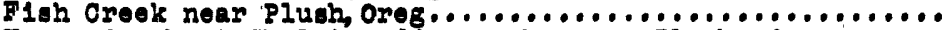
Honey oreok at Ohalwtrand ranoh, near Pluah, oreg....... Boney Oreok near Plush, Oref. +1000 ; 1810-15;

Twelvem1le Oreok near Plush, Orog................

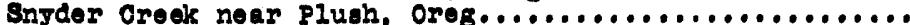
Pelloan Lake near Adel, Oreg........................

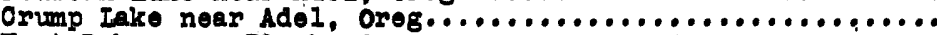
Hart Lake near Plush, Oreg..........................

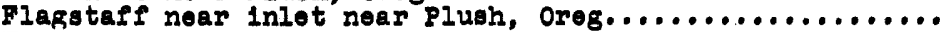
Flagstaff Lake near Pluah, oreg..................... Lower Campbell Lake near Plush, Oreg................. Stone Oorral Lake noar Plush, oreg.................. Bluejolnt Lake near Plush, oreg....................

Abert Lake near Valley Falls, Oreg................... Chowaucan River at dam sito near Palsloj, oreg.......... Chowaucan River near Palsley (above M1li Creek), Oreg... Chewaucan Rlver above Conn ditch, near Palsloj, oreg....

$\begin{array}{rl}-\overline{58} & 1915 ; 1917-18 . \\ \overline{-} & 1912-16 . \\ \ddagger 275 & 1912-21 . \\ & 1912 ; 1924-\end{array}$

\footnotetext{
* Only gage heights or discharge measurements, or both. $\mp$ Revised.

t In reports of state Ingineer of Oregon.
} 
Abert Lake Basin--Continued.

Chewaucan River at Paisles.

Chewaucan River at Narrows, near Paisley, oreg..........

Chewaucan River at Hotchkiss Ford, near Palsley, Oreg...

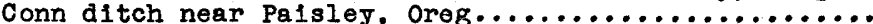
Smalls Creek at Palsley, Oreg.................... Jones-Innis-zX ditch near Paisley, Oreg.............. Crooked Creek near valley Falls, oreg...............

SUMMER LAKE BASIN

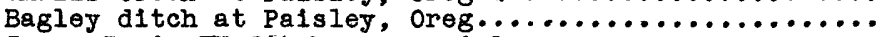

Drainage area (square miles) $\$ 280$

Ana River near Summer Lake, oreg...................

\section{SILVER IAKE BASIN}

Silver Lake near Silver Lake, Oreg...................

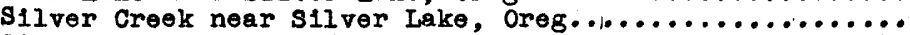
Silver Lake inlet near silver Lake, oreg.............. West Fork of S1lver Creek near Silver Lake, Oregip.....

silver Lake Irrigation District Canal near silver Lake

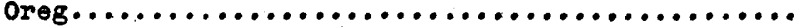

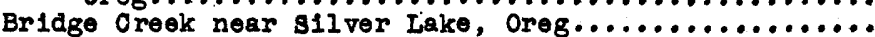

Buck Creek near silver Lake, Oreg................. Duncan Creek near s1lver Lake, Oreg...................

\section{MALHEUR AND HAFNEY ILAKRS BASIN}

Malhour Lake Basin:

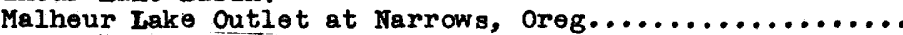

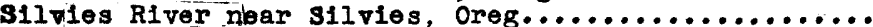

S1lvies River near Burns, Oreg................... Weat Fork Silvies River near Lawen, Oreg.............. Bagt Fork silvies River near Lawen, oreg............

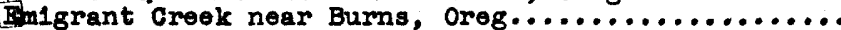

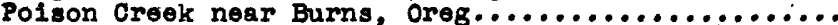

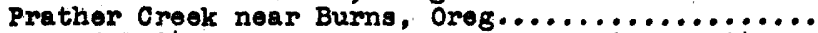
Donner und Blitzen River near Frenchglen (Diamond) Oreg.

Donner und Blitzen River near Narrows, Oreg........... Donner und Blitzen R1ver near Voltage, Oreg...........

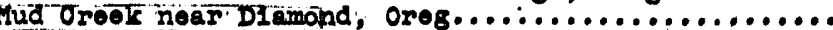
Bridgo Creok near Frenohglen (Dlamond), Oreg......

Krumbo Oreak near Dlamond, oreg.. ..............

Buena V1ata Canal noar Narrows, Oreg................

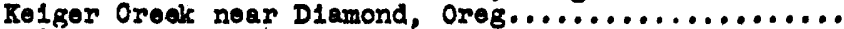

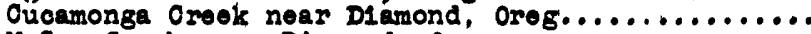

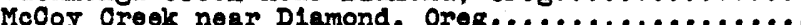

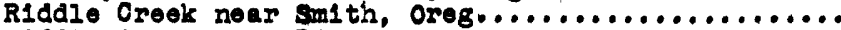

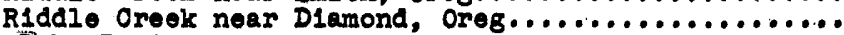
Harney Dake Bas In:

Mud Lake Outlet near Narrows, Oreg...................

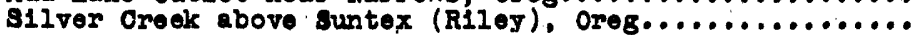

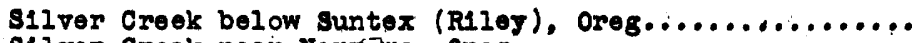

S1Iver Creek near Narrows, Oreg...................... Chlokahominy Creok near Buntex, orog...............

Rock Quarry Crook near Suntex, orog.................

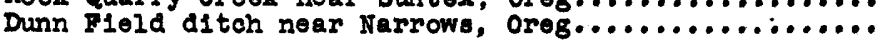

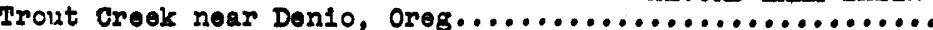
Ittle Cottonwood Creok nesr Denio, oreg.................

Van Horn Oreok near Denlo, Oreg......................

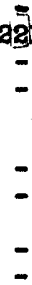

\section{ALVORD LAKS BASIN}

\section{TUMIUM IAKE BASIN}

$\overline{450}$

934

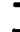

Period of record 1905-7; 1909-12; 1913. $1914-21$.

$1914-21$.

1914-20.

1914-21.

1914-20.

1914-21.

$1912-13$.

$1904-6 * 1909-10 * \%$ (1914-15*; 1917-30*1930-36 t.

1914-17; 1921.

$1904-7$; $1909-$

1922 .

1919-23; 1925-28;

$(1929-32) \mathrm{t}$.

1922-28; $1929-41$.

1905-6; 1910-12;

$1922-23$.

$1905-6$; 1909-11; 1919-23.

$1922-23$.

1903-6; 1911-i3; 1914 ; 1916. 1903-5; 1909-12; 1916; 1921-23. 1903-6; 1908 $1916-17$; 1919 ; 1922.

1916.

1921 .

$1921-22$.

1921-23:

1909-21; $(1829-30) \mathbb{t}_{;}$1937-

1916-20:

$1916-19 ; 1921-28 ; 1938-46$.

1911-16, (1930)

1811; $1012-16$; 1937 -

1911; 1913; (1930) Yt.

1915-20.

1909-13; 1816-21; (1930), 1911-13; 1916; (1830) t? 1909-14; 1916-21; (1930) 1811.

1917-21.

1916-18; 1981-22. $1904-6 ; 1909=18 ; 1914-23$; $(1926-26) t$

1812-15; 1917; 1919; 1921-23. 1917-23.

1917; (1981) 나 1922-23.

(1921) E; 1922.

1917.

$1911-12$, 1922-23; 19251811-12.

1911.

\footnotetext{
\$Rev1sed.

* Only gage holghta or d1soharge moasurements, or both.

$t$ In reports of gtate Englneer of Oregon.
} 


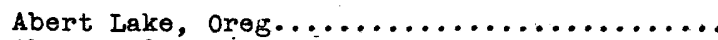

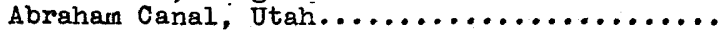

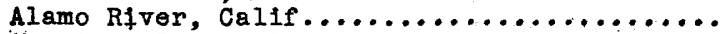

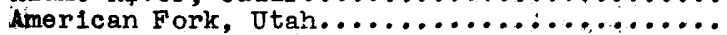
Amerlcan Fork, South Fork, Utah...........

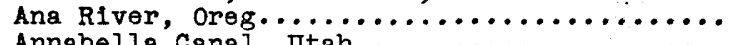

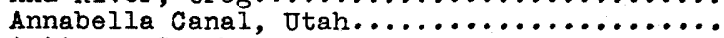
Antimony Creek, otah................... A. O. Canal. See Collins Canal.

Ash Creek, Calif...................... Asay Creok, Utah...................

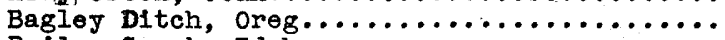

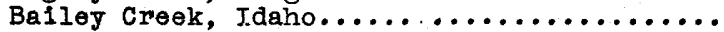
Bailey Creek, diversions from, Idaho........

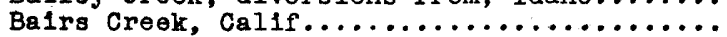

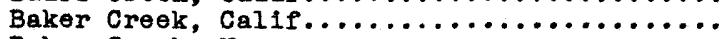

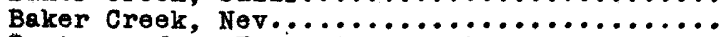

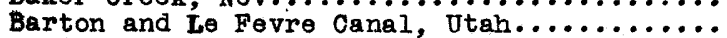

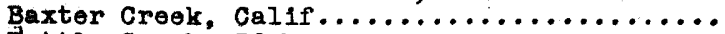
Aattle Creek, Idaho. .................

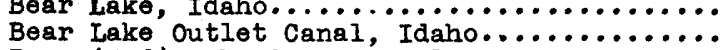

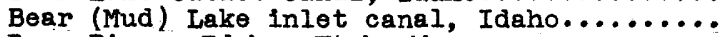

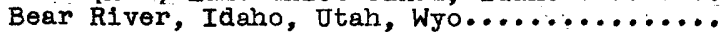
Bear River, diversions from, Idaho, Utah, Wyo Bear River tributaries, diversions from, Utah

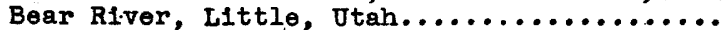
Bear River, Little, diversions from, Utah.... Bear River, Iittie, East Fork, Utah.........

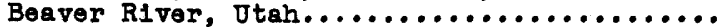
Bennington Oreok, Idaho.................

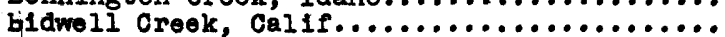
B1g Cottonwood Oreok, Dtah............. B1 Oreek, Nev......................

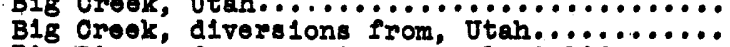
B18 PIne and Owens RIver Canal, Oalif....... B1g P1ne areok, Oal1f..................... B1g Warm springs, Nov . . . . . . . . . . . . Biroh Crook, Callf................... Elich orook, Nov....................... Biroh areok, ttah......................

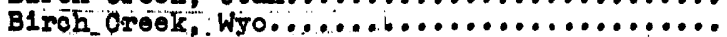
Blshop Crook, Calif...................

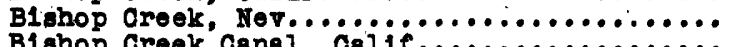
Blackmith Fork, Jtah................. Blacksm1th Fork, diversions from, Utah...... Hyrum City Power Canal. Bloomington Croek, Idaho................... Bluejolnt Iako, oreg................... Boumno (Goorge), Wro .................. Box Danyon Oreek, dal1f.................. Box BIder Oreok, Utah, .................. BrIdge Oreok, Oreg. (MaIhour and Harney

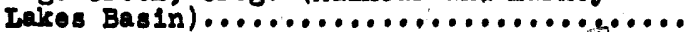

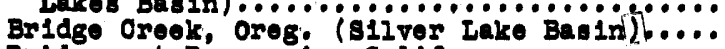
Bridgeport Reservolr, Callf.............. Brooklyn Danal, Jtah.................. Buck Creok, oreg. $\ldots \ldots \ldots \ldots \ldots \ldots \ldots \ldots \ldots \ldots$ Bulilon Oreok, Jtah. See Pino creok. Buena vista oanal, orog................. Campbell Lake, Lower, Oreg.............. Canal $A$, Jtah...................... Canal $B$, Jtah.......................

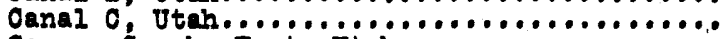

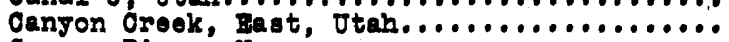
Carson R1ver, Nov..................... Carson River, Bast Fork, dailf., Nev........

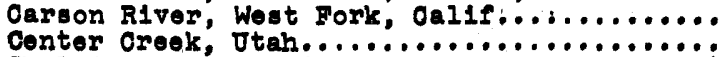
Chalk orook, otah (tributary to Wober Rivor)
13 Chalk Creek, Utah (Pavant Valley)........

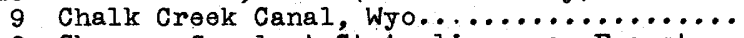

9. Chapman Canal at State Iine near Evanston.

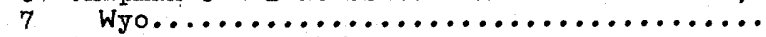

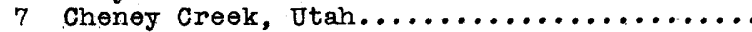

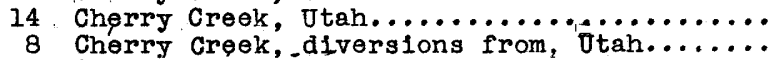

8 Chewaucan River, Oreg..................13-14

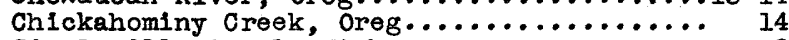

10 Cirelevilie Canal, Utah................ 8

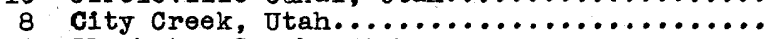

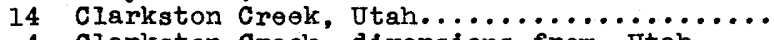

4 Clarkston Creek, diversions from, Utah.....

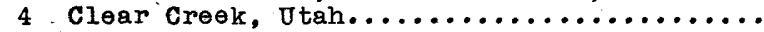

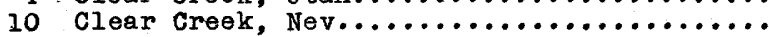

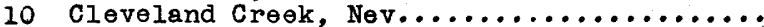

9 Cleveland Irrigation Co. Canal, Idaho......

8 Coal Creek, utah......................

13 Collins (George) Canal, Calif.............

5 Collins (A. 0.) Canal, Calif.............

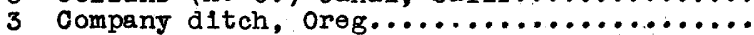

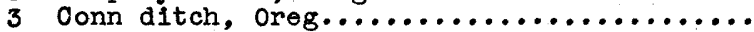

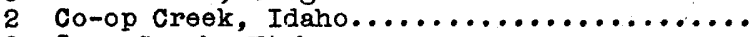

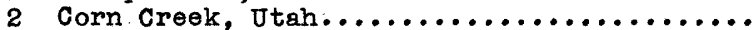

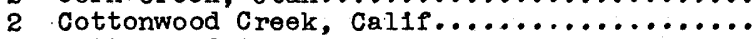

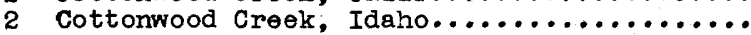

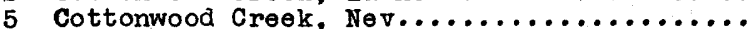

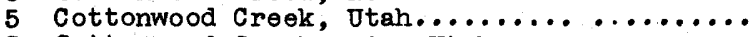

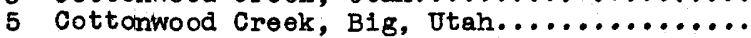

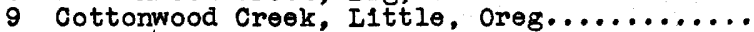

4 Cottonwood Creek, Iittle, Utah............

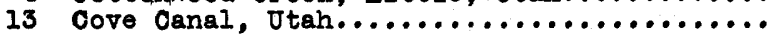

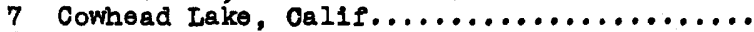

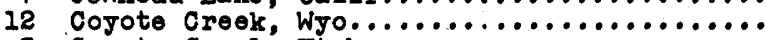

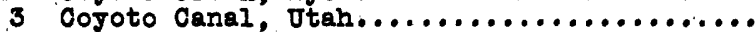

3 Crane Greek, oreg.....................

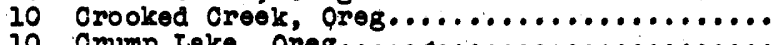

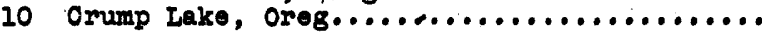

9 Cub River Irrigation Oo's. Pump Canal, Idaho

I0 Oub River-Worm Oreek Canal, Idaho.........

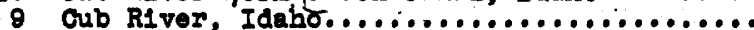

5 Oub R1ver Canal; Idaho..................

3 Cucamonga Croek, oreg ..................

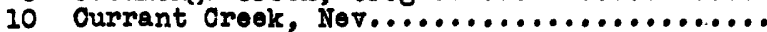

12 Cutler Reservoir, Utah.................

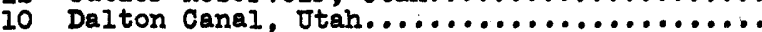

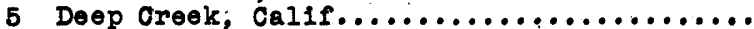

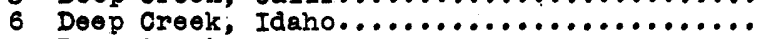

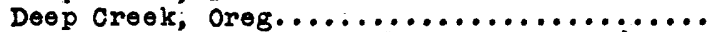

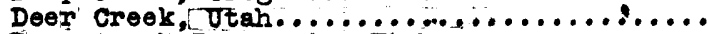

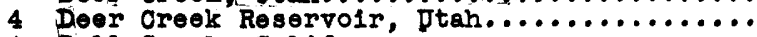

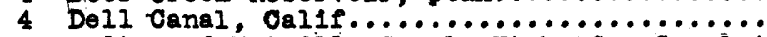

13 Delta and Melvilio Canal, Utah. See Canal A

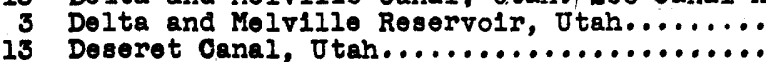

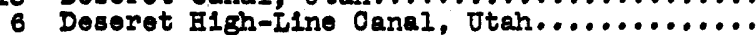

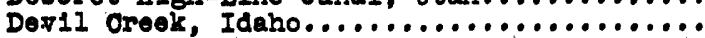

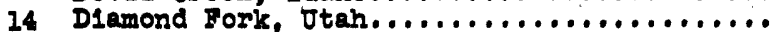

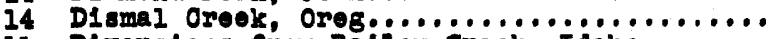
11 Diverelon from Balloy Crook, Idaho........ 8 Diveralons from Boar River, Idaho, Utah, Wyo 14 Diversion from Boar RIvor tributarles, Otah 18 Diversions from Bear River, Iittio, Jtah... Divera ions from B1g crook, Jtah........... 14 Diversions from Blaoksmith Fork, Jtah....... 13 Diversion from Bloomington oreok, Idaho... 13 Diversions from oherry drook, Utah......... (9) Diversion from Olarketon Oreok, Jtah....... 9 D1veralon from Bightmilo Creek, Ideho...... - Divera1ons from Bmigration Creok, Idaho.... - Diveralons from Plah Haven Crook, Utah..... 11 Diversions from Formation springs, Idaho.... 11 Diversions from Georgetown Creek, Idaho.... 11 Diversions from Flgh Groek, Utah.......... 9 Diversions from Logan RIver, Jtah........... 


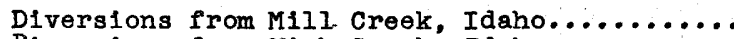

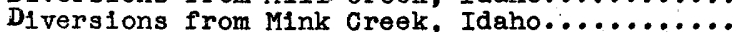
Diversions from Montpelier Creek, Idaho...... Diversions from North Creek, Idaho............ Diversions from Otter Creek, Utah, tributary

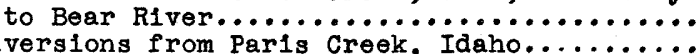
Diversions from Randolph Creok, Utah.......... Diversions from st. Charles Creek, Idaho......

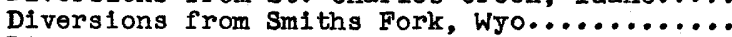
Diversions from Soda springs, Idaho.......... Diversions from Sulphur Creek and Willow

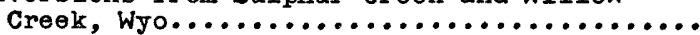

Diversions from Summit Creek, Utah.......... Diversions from Swan Creek, Utah............ Diversions from Thomas Fork, Idaho $\ldots \ldots \ldots \ldots . . . . .$.

Diversions from Trout Creek, Idaho...........

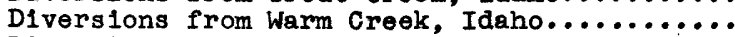
Diversions from Weston Creok, Idaho.......... Diversions from Whiskey Creok, Idaho......... Diversions from Woodruff creek, Utah.......... Diversions from Yellow Creek, Wyo...........

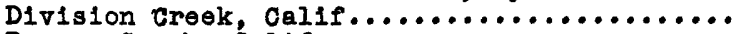

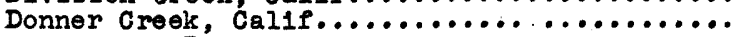
Donner und Biltzen River, Oreg..d.......... Dover Canal, Utah......................

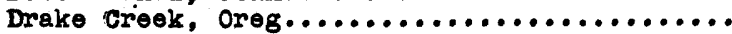
Dry Creok, Utah.......................

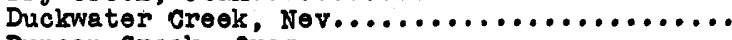

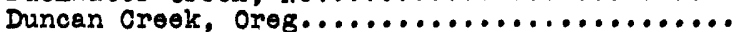
Dunn Field ditch, oreg...................

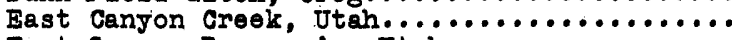

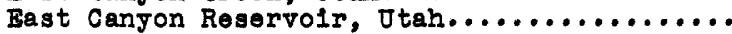
East Fork. Soe name of main stroam.

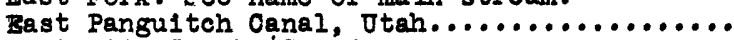

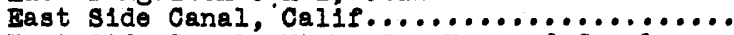

East Side Canal, Utah. Seo Hammond Canal.

East Walker River, Calif., Nev..............

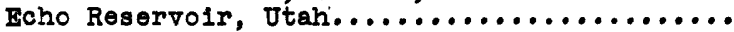

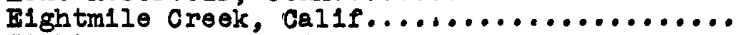

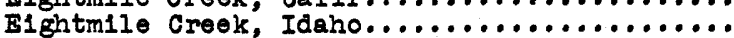

Elghtmile Creek, diversions from, Idaho......

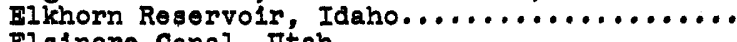

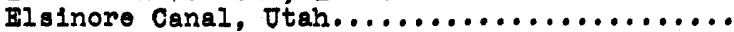
Bm1grant Creok, oreg....................

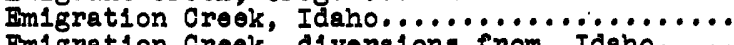
Bmigration Creok, diversions from, Idaho.....

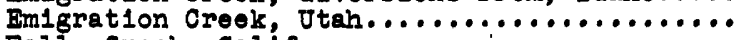
Falis Creok, Calif ....................

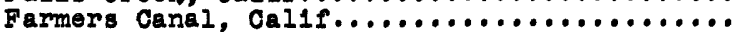

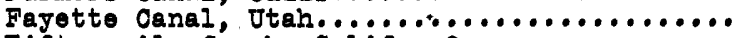
Fifteonm1le Crook, Calif., oreg..............

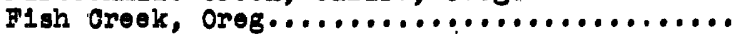
Fish Fatchery springs, Utah..............

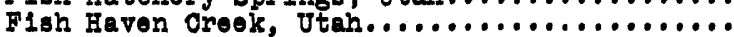
Flin Haven Oreok, diversions from. Utah...... Flagstaff take, oreg..................

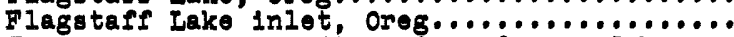
Fomation Springs, diversions from, Idahd.... Fort Croek, $t_{a h} \ldots \ldots \ldots \ldots \ldots \ldots \ldots \ldots \ldots \ldots \ldots \ldots$

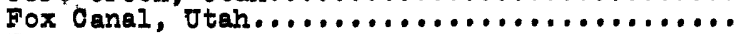

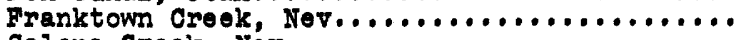

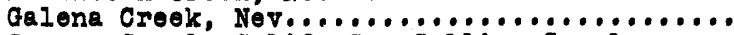
Goorgo Oanel, Calif. Seo Collins Canal.

George Creok, Oalif....................

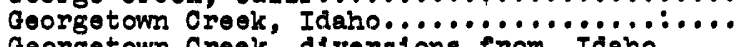
Georgetown Creek, diversions from, Idaho..... Gold Run Crook, dal1f.................... Goodalo Creok, Callf..................... Great Salt Lake; Utah..................

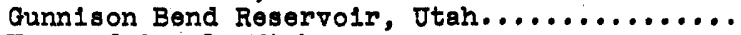

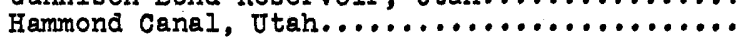

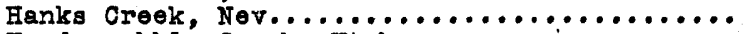
Hardscrabble Creek, Jtah.................. Hatch Bonch Canal, Jtah..................

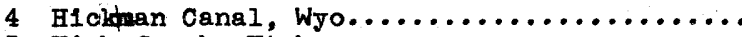

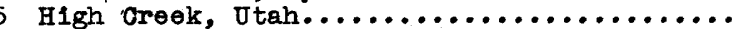

3 High Creek, diversions from, Utah.........

4 Hillside (North) Canal, Calif............

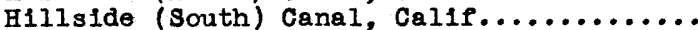

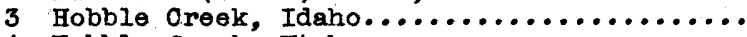

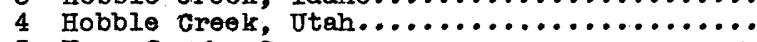

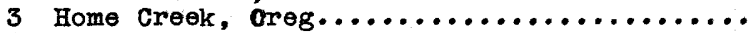

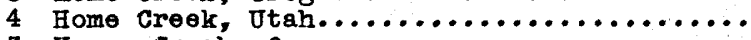

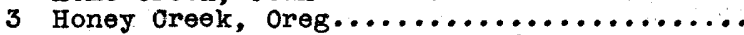

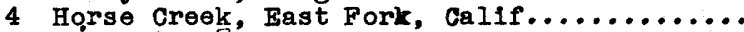

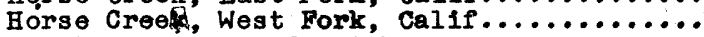

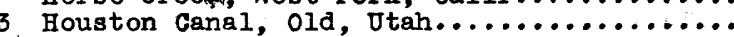

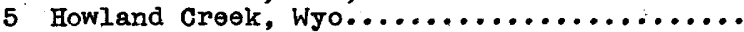
4 Humboldt-Lovelock Irrigation, Light \& Power

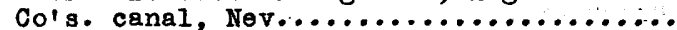

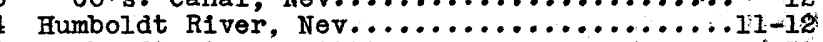

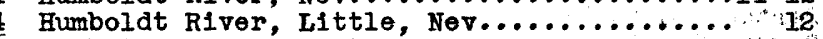
5 Humboldt River, North Fork, Nev.......... 12

4 Humboldt River, South Fork, Nev............ b-12

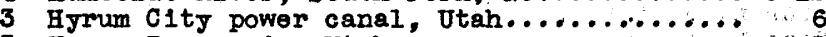

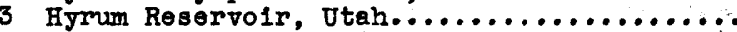

10 Independence Oreok (Pyramid and Winnemucca

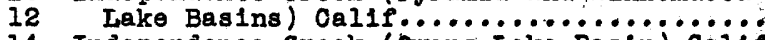
Independence Creek (Owens Lake Basin), Calif Seo Littlo Pine creok.

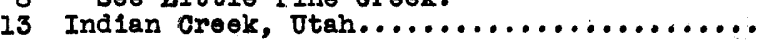
Innis ditch. Seo Jones-Innis-zX.

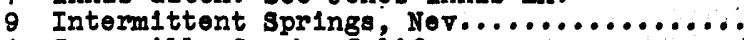

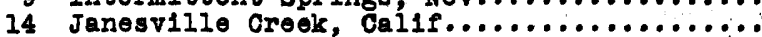

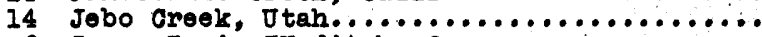

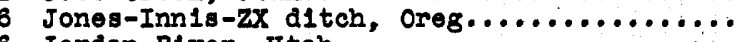

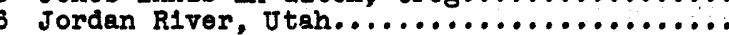

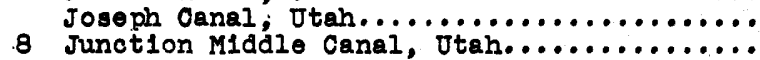

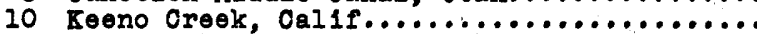

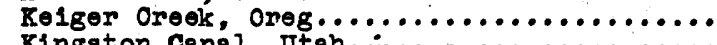
11 Kingaton Canal, vtah. $\ldots \ldots \ldots \ldots \ldots \ldots \ldots \ldots \ldots \ldots$

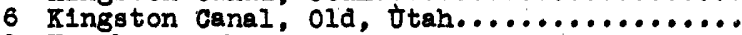

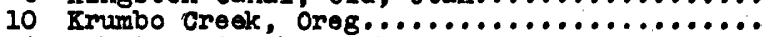

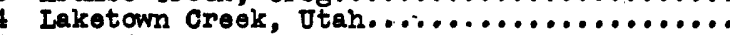
4 Lake Tahoo, Caí1f......................

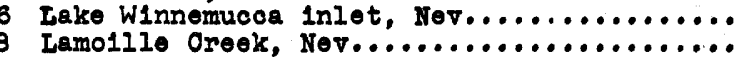

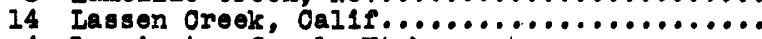
Leamington Canal, Jtah...............

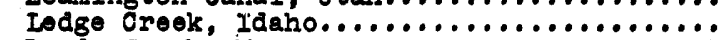

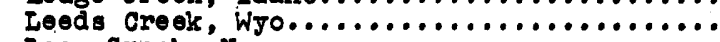

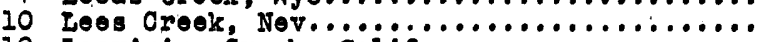

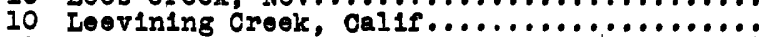

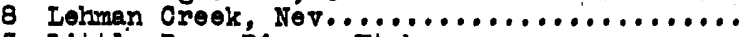
13 Ilttle Bear River, Utah............... 13 Iltile Bear R1ver, Blast Fork, Utah......... 3 I1ttlo Cottonwood oreok, oreg............

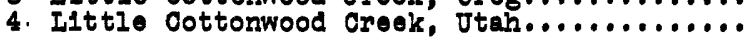
4 Ilttlo Creok, Idaho.................. 13 Ittle Humboldt RIver, Nev............. 13 Iltle Malad R1vor, Idaho............... 4 Little Plne Greok, oal1f...............

7 I1ttle Trucke R1ver, Cal1f............

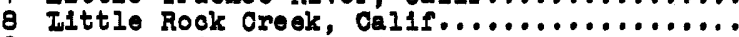

12 Logan, Hydo Park, and Smithriold Canal, Jtah Logan Northern Oanal, Jtah..............

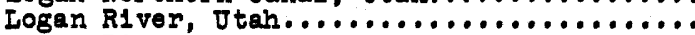
Logan River, diversions from, Utah......... Lone Plne Oreek, cal1f..................

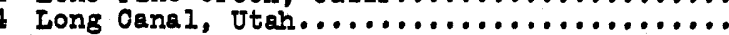

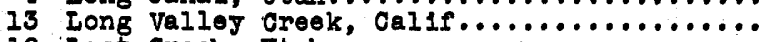

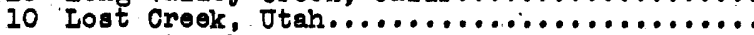

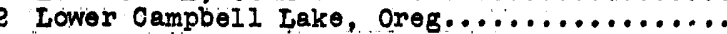

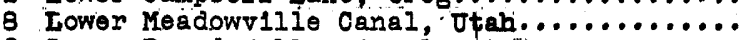

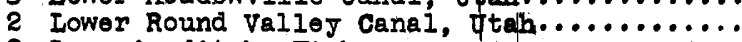

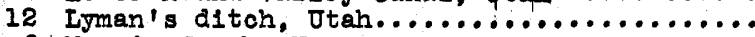

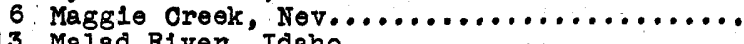

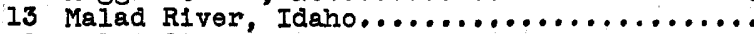
8 Melad Rlver; Ilttle, Idaho.............. 


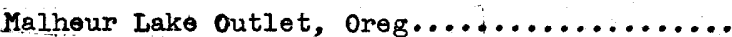

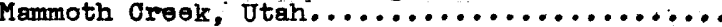

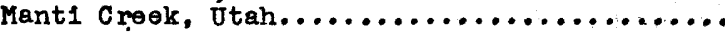

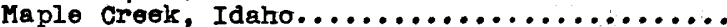
Maple Creek, diversions from, Idaho......... Maple Creek, Jtah....................

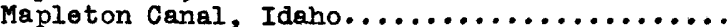

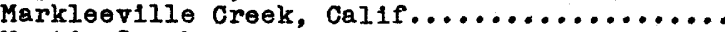

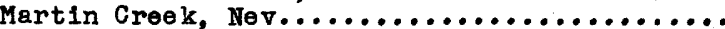

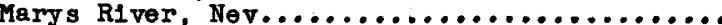
McCoy Oreek, oreg.....................

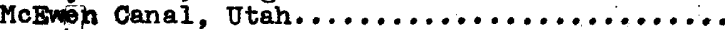

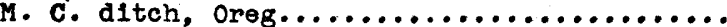

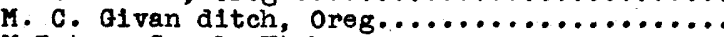

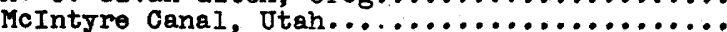
McNally Canal, Callf.................... Meadow Creek, Otah....................

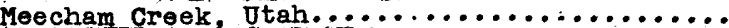

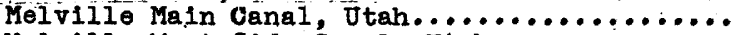
Melville West. Side Canal, Utah................

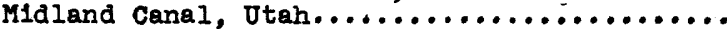

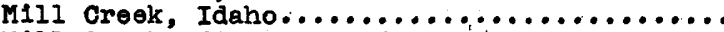
Mill Creok, diversions from, Idaho............ Mill Creok, Utah (tributary to Great Balt

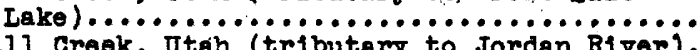
M111 Greek, Utah (tributary to Jordan River). M11l Creek, Wyo......e.e.e.eee............. Mill Groek, diversions from, wro............

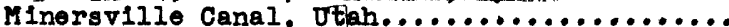

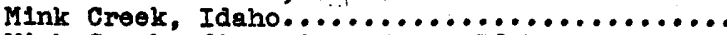
Mink Creek, diversions from, Idaho.............

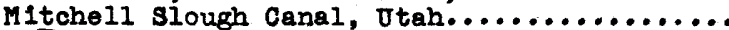

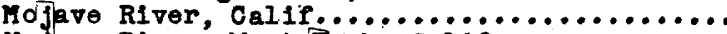

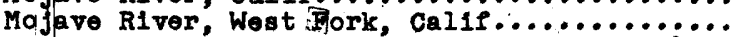

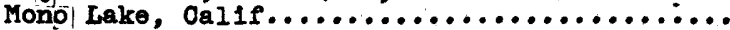

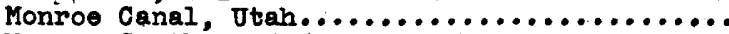

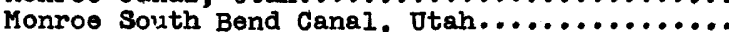

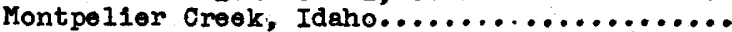
Montpelier Creek, diversions from, Idaho..... Mud Creok, Oreg. (Malhour and Harney Lakes

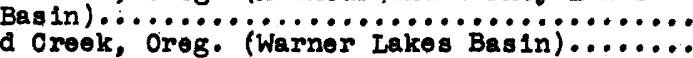
Mud Croek, Oreg. (Warner Lakes Basin) ....... inlot ounal.

Mrd Lake outlet, oreg...................

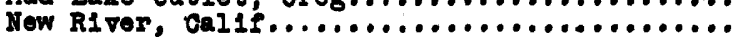
North Oanal, Calif. Soo H1lisido Oanal.

North Crook, Idaho .................... North Creek, diversions from, Idaho.......... North Croek, North Fork, Jtah.............. North Orook, South Fork, Utah............... North Fork. Beo name of main stream....... Oak orook, oal11...................... Ogden Rivor, Utah...................... Ogden River, Middlo Fork, Dtah............. Ogden R1vor, south Fork, Jtah.............. old Bear Lake outlet, Idaho................

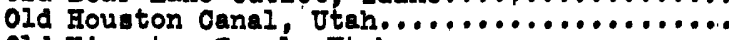
old Kingston Canal, Jtah................ One1da Revervo1r, Idaho.................... otiter Oreok, Utah (tributary to Boar River)... Otter oreok, diversions from (tributary to

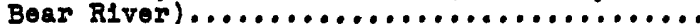
Otter Creok, Middle Fork, utah.............

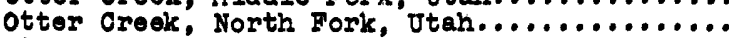
Otter Oreok, South Fork, Jtah.............. Otter Oreok, Utah (tributary to Fast Fork

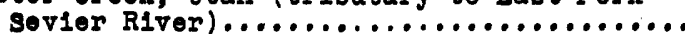

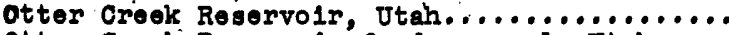
Otter Creok Reservolr feeder canal, Utah...... Overland Creok, Nev.....................

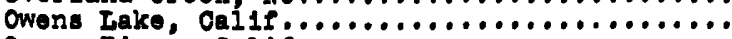

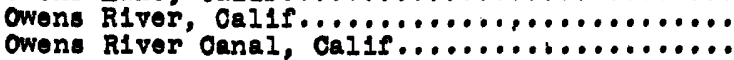

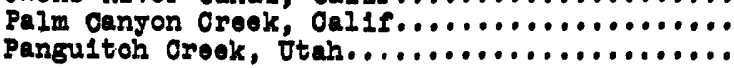

14 Paris Greek, Idaho................... Paris Creek, diversions from, Idaho.........

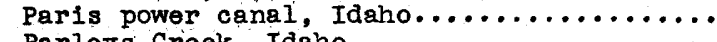

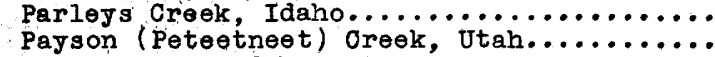

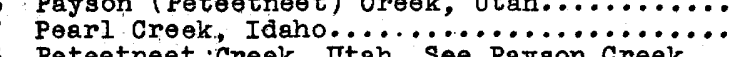
Peteetneet Greek, Utah. See Payson Creek...

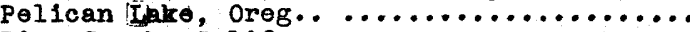

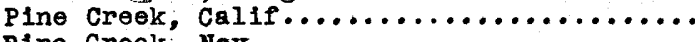

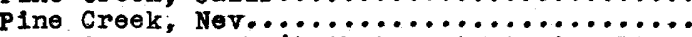
Pine Creek, Utah (tributary to Sevier River) Pine Creek, Jtah (Pavant Valley)...........

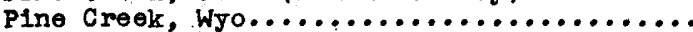

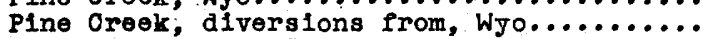

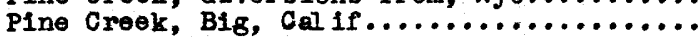

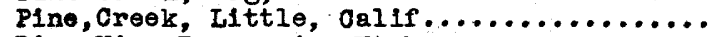

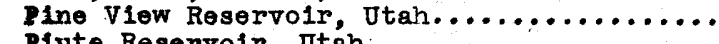

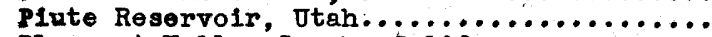

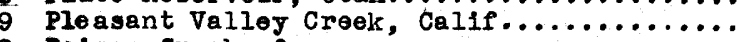
9 Poison Creek, oreg $\ldots \ldots \ldots \ldots \ldots \ldots \ldots \ldots \ldots \ldots$

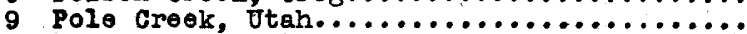

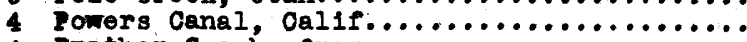

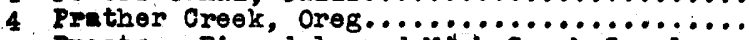

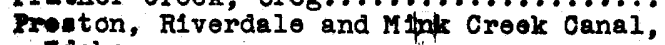

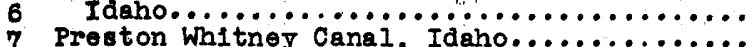

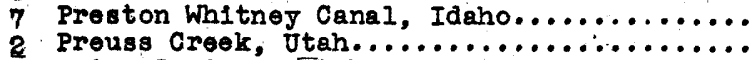

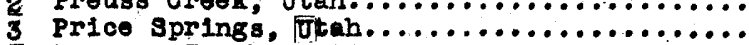

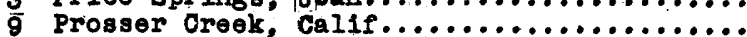

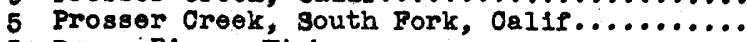
5 Provo River, Utah.....................

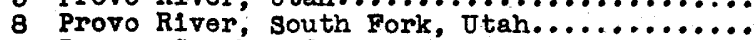

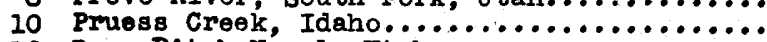

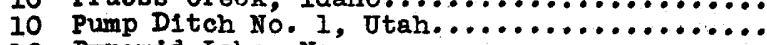

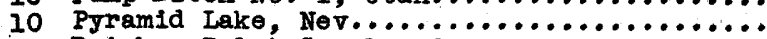

8 Rainbow Inlot Canal, Idaho..............

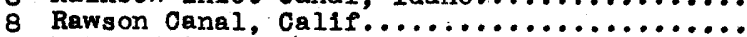

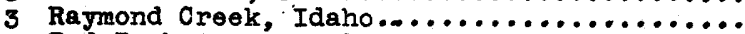

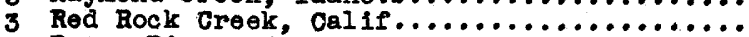

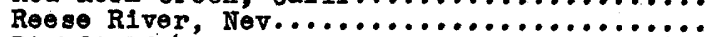

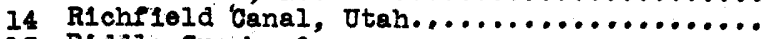

13 Ridale Creek, oreg. ..................

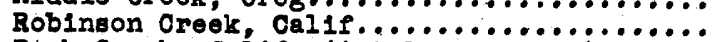
nock Crook, Oalif. (Antolope vailioy).........

14 Rock Crook, Cal1f, (Owens Lake Bas inj....... hook Crook Cal1f. (Warner Lakes Basin).....

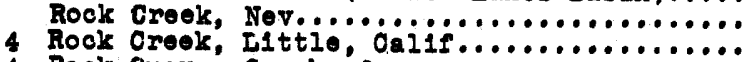
4 Rock Quarry Creok, oreg..................

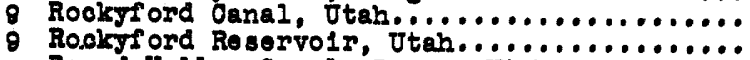
Round Valley Canal, Lower, Utah.............

10 Round Valley Oanal; Jpper, Jtah...........

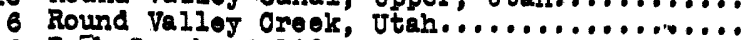
\& Ruge Crook, cal1f.....................

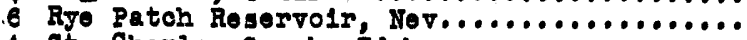

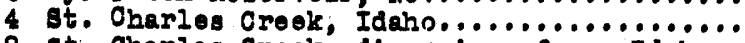
8 St. Charlos Creok, diversions from, Idaho...

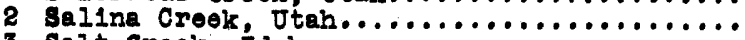

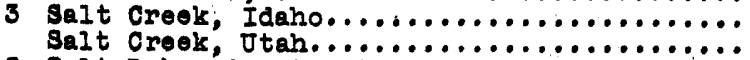

3 Salt Lake, Great, Utah..................

3 salton sea, cal1f $3 \ldots \ldots \ldots \ldots \ldots \ldots \ldots \ldots \ldots \ldots \ldots$

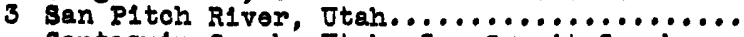
Santaquin Creok, Utah. See summit Creok.

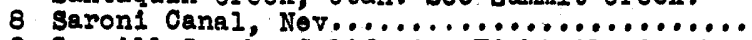

8 Sawm1li Crook, Calif. Soo Fightmilo Crook.

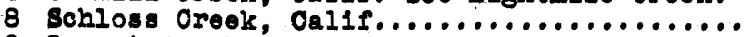

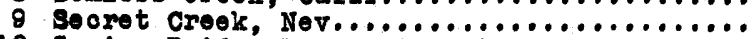

10 Sevier Bridgo Reservoir, Jtah...............

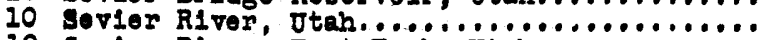

10 sev1er R1vor, Bast Fork, Jteh............

10 Sevier R1ver Iand Wator Co's. bypass, Utah 8 sevier River land \& Water co's. Oanal, Jtah
4

4 
Stream or Reservoir

Sevier River Land \& Water Co's. reservolr

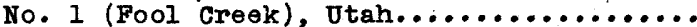

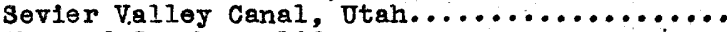

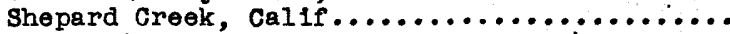

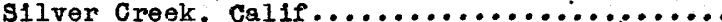
Silver Creek, Oreg. (Malheur and Harney Lakes

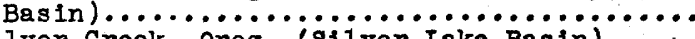
Silver Creek, Oreg. (Silver Lake Basin)......

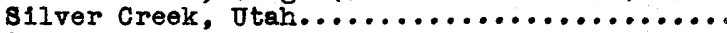

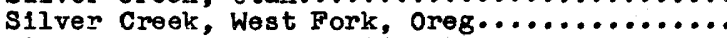

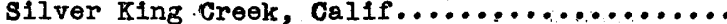

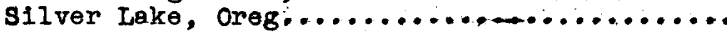

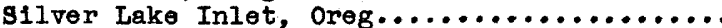
Silver Lake Irrigation District Canal, Oreg..

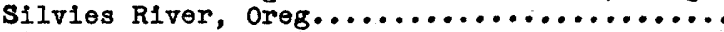

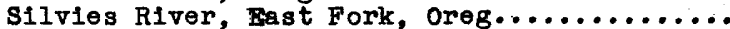

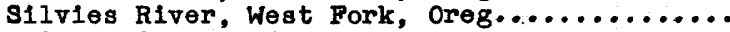

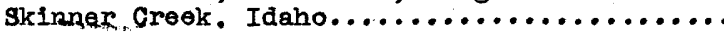

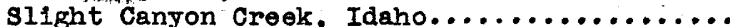

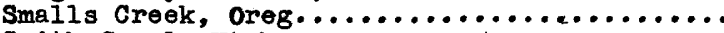

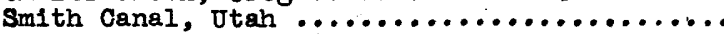

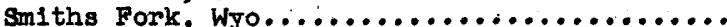

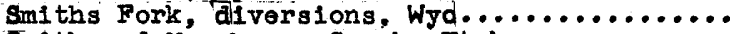

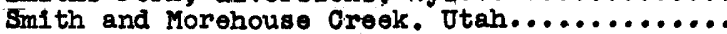

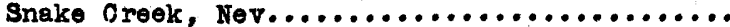

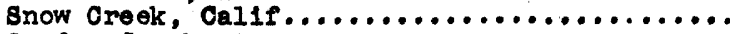

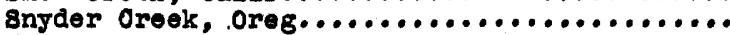
Soda Creok, Idaho....................... Soda Creok, diversions from, Idaho........... Soda Reservo1r, Idaho..................... South Oanal, Cal1f. Soe H1llside Canal........

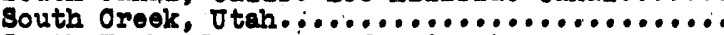
South Fork. Seo name of main stream.

Southern Panifle Cola. ditch, Cal1f.......... Spanlah Fork, Utah......................

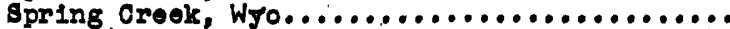
Spring Oreek danal, Idaho.................

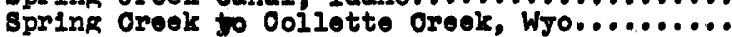
Starr Grook, Fev....................... State Canal, Uțah (5 stationa) .............. Stauffer Oreek, Idaho......................

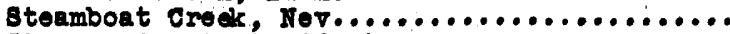
Stevens Ganal, dalif.................... Stone Corral Lake, Oreg................... Strawberry tuniel, utah. ................

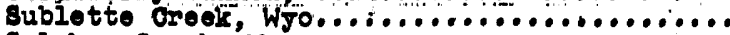

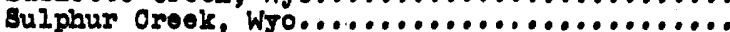
Sulphur Creok, divers 1ons from. Wyo.......... Summit Orook. "Dtah (tributary' to Boar River). Surmit Oroek, Utah (tributary to Jtah Lake).. Gummit Croek, Jtah...................... Surmit Oreek, diversions from, Jtah........... Surplus Canal, Utah..................... Susan R1ver, dal1f..................... Swager oreek cal1f..$\ldots \ldots \ldots \ldots \ldots \ldots \ldots \ldots \ldots \ldots$

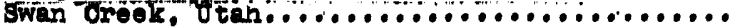

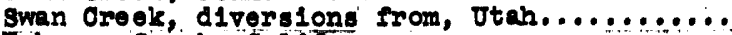

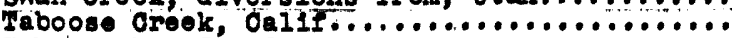

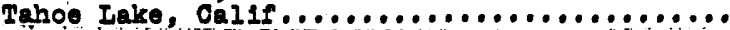

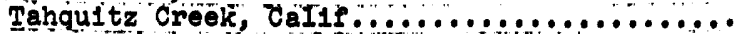

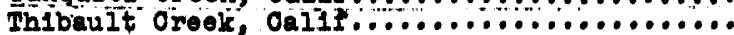
Th1rd Creok, Idaho.....................

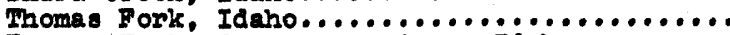
Thomes Fork, diversions from, Idaho......... Thousand springs Oreok, Nev................ Throe Creoks, otah.....................

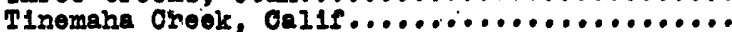
Topaz Reservolí, oalif...................

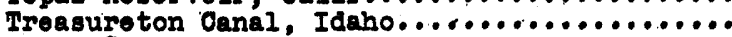
Trout Oreek, Oreg....................... Trout Oroek, Idaho.....................

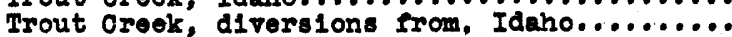
Truckee Rlver, Callf., Nov.................

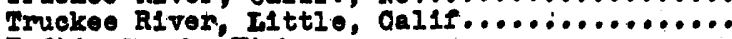

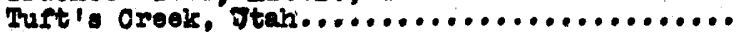

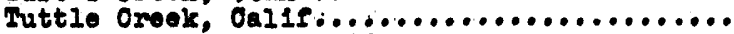
Twelvemlile oreek, dalif................. Twelvem1lo Oreok, ores..................
Page

Stream or Reservoir

Page

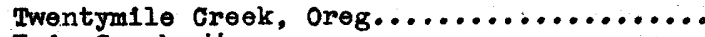

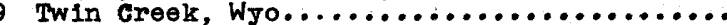

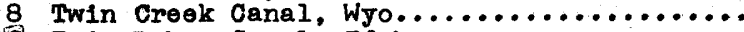

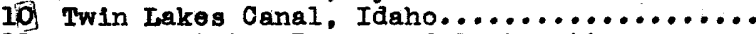

11 United states Bureau of Reclamation power

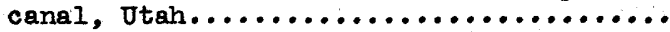

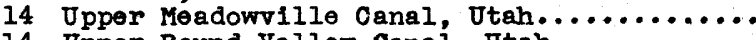

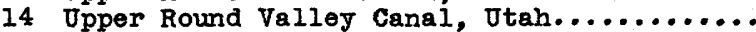

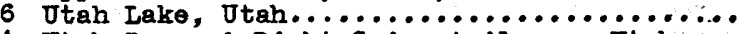

14 Utah Power \& Iight Co's. tallrace, Jtah....

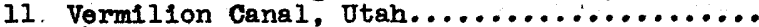

14 Van Horn Greek, oreg..................

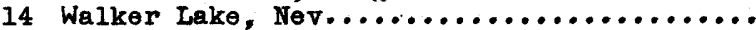

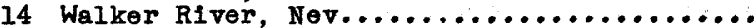

14 Walker RIver, East; Calif., Nev...........

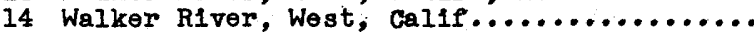

14 Walker River, West, East Fork, Cal1f.......

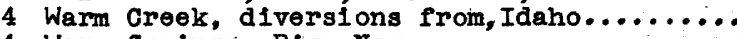

4 Warm Springs, Blg, Nev..................

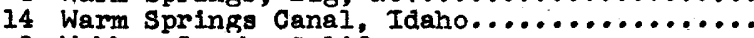

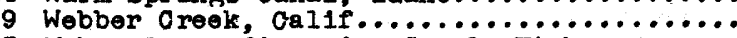

3 Weber-Provo diversion Canal, Utah..........

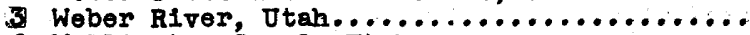

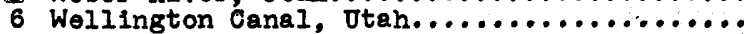

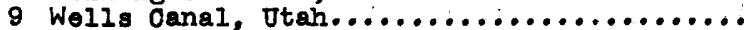

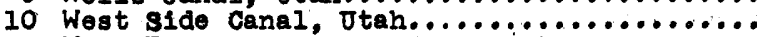

13 West Fork. Soe name of main stream.

4 West Fork, Idaho......................

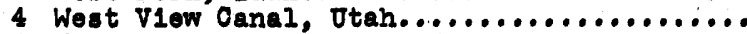

4 Weat Walker River, Calif., Nov............

4 West Walker River, East Fork, Calif.........

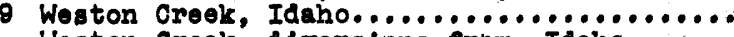
Weston Creok, diversions from, Idaho........

10 Wh1skey Oreok, diversions from.............

7 Will lams Orook; Idaho.....................

3 Williams Creok, diverglons from, Idaho.....

4 Willow Oreok, Cal1f.....................

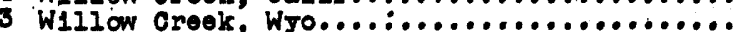

12 Wizlow Oreok, diversions from, Wro........

8 Winnemucos, Inlet, Iake, Nev..............

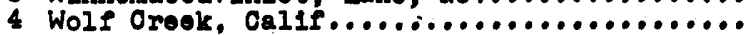

12 Woodruef Creok, Jtah...................

+ I0 Woodruff Groek, South Fork, Jtah............

13 Woodruef Creoky divorsions erom, Utah.......

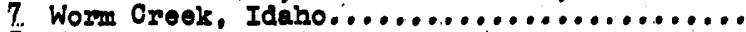
3 WrIght Oroek, Idaho ....................

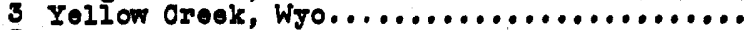
3 Yellow Creek, diversion from, Wyo........ $5 \mathrm{ZX}$ ditoh. Soe Jpnes-Innis-zX.
13

3

5

7

3

5

14

11

11

11

11

1.1

4

6

12

6

8

2

4

11

11

5

4

5

13
5

5

13

11

4

10

12

10

10

3

3

9

10

11

5
14

14 